\title{
Analysis of the noise-induced bursting-spiking transition in a pancreatic beta-cell model
}

Aguirre, J.; Mosekilde, Erik; Sanjuan, M.A.F.

Published in:

Physical Review E. Statistical, Nonlinear, and Soft Matter Physics

Link to article, DOI:

10.1103/PhysRevE.69.041910

Publication date:

2004

Document Version

Publisher's PDF, also known as Version of record

Link back to DTU Orbit

Citation (APA):

Aguirre, J., Mosekilde, E., \& Sanjuan, M. A. F. (2004). Analysis of the noise-induced bursting-spiking transition in a pancreatic beta-cell model. Physical Review E. Statistical, Nonlinear, and Soft Matter Physics, 69(4), 041910. https://doi.org/10.1103/PhysRevE.69.041910

\section{General rights}

Copyright and moral rights for the publications made accessible in the public portal are retained by the authors and/or other copyright owners and it is a condition of accessing publications that users recognise and abide by the legal requirements associated with these rights.

- Users may download and print one copy of any publication from the public portal for the purpose of private study or research.

- You may not further distribute the material or use it for any profit-making activity or commercial gain

- You may freely distribute the URL identifying the publication in the public portal 


\title{
Analysis of the noise-induced bursting-spiking transition in a pancreatic $\beta$-cell model
}

\author{
Jacobo Aguirre, ${ }^{1}$ Erik Mosekilde, ${ }^{2}$ and Miguel A. F. Sanjuán ${ }^{1}$ \\ ${ }^{1}$ Nonlinear Dynamics and Chaos Group, Departamento de Matemáticas y Física Aplicadas y Ciencias de la Naturaleza, Universidad Rey \\ Juan Carlos, Tulipán s/n, 28933 Móstoles, Madrid, Spain \\ ${ }^{2}$ Department of Physics, Technical University of Denmark, DK 2800 Lyngby, Denmark
}

(Received 15 September 2003; published 30 April 2004)

\begin{abstract}
A stochastic model of the electrophysiological behavior of the pancreatic $\beta$ cell is studied, as a paradigmatic example of a bursting biological cell embedded in a noisy environment. The analysis is focused on the distortion that a growing noise causes to the basic properties of the membrane potential signals, such as their periodic or chaotic nature, and their bursting or spiking behavior. We present effective computational tools to obtain as much information as possible from these signals, and we suggest that the methods could be applied to real time series. Finally, a universal dependence of the main characteristics of the membrane potential on the size of the considered cell cluster is presented.
\end{abstract}

DOI: 10.1103/PhysRevE.69.041910

PACS number(s): 87.17.- d, 05.45.-a

\section{INTRODUCTION}

The pancreatic $\beta$ cells are responsible for the secretion of insulin, the hormone that regulates the glucose concentration in the blood. Autoimmune destruction of $\beta$ cells results in type-I diabetes, and this illness, if not treated correctly, can cause the death of the patient.

The electrophysiological behavior of pancreatic $\beta$ cells shows bursts in the membrane potentials for intact islets of Langerhans, where the cells are coupled in groups of 1000-10 000 cells. These bursts consist of the alternation of active phases and silent phases. During the active phase, the membrane potential oscillates rapidly, while it rests almost unchanged during the silent phase. Besides $\beta$ cells, many other cell types show bursting electrical activity [1]. The bursting activity can be quantified by means of the so-called plateau fraction, that is, the ratio of time that the cell spends in the active phase over the total recording time. This quantity seems to be physiologically very relevant, as several experimental reports [2-4] have concluded that there is a direct correlation between the plateau fraction and the rate of insulin release. The main reason is that the intracellular calcium concentration $\left[\mathrm{Ca}^{2+}\right]_{i}$ increases during the active phase, andit is generally accepted that calcium has a key role in the secretion of insulin. The synchronization between the oscillations of $\left[\mathrm{Ca}^{2+}\right]_{i}$ and the active-silent alternation of the electrical activity has been thoroughly studied by Santos et al. [5]

The bursting electrical activity in intact islets is often synchronized, presumably via gap junctional coupling [6-9]. However, the bursts get distorted when we consider the membrane voltage of smaller clusters, and about one third of the isolated $\beta$ cells only show stochastically distributed spikes [10]. A complete explanation is still lacking of this difference in behavior between coupled and isolated cells, although several possible answers have been proposed. An obvious reason could be that the cells change their behavior when removed from their normal environment. This is a common phenomenon in cellular biology and an obstacle to much experimental work. On the other hand, Sherman et al.
[11] and Chay and Kang [12] proposed the stochasticity associated with the random opening and closing of certain ion channels to be the main reason for this peculiar behavior. In coupled cells inside intact islets, the noise would be distributed among the membrane of many different cells and therefore its influence would be smaller, letting the bursting behavior appear. This explanation is commonly known as the channel-sharing hypothesis. However, recent experimental results on single-cell electrical behavior have shown that the situation is not so simple. In fact, it was demonstrated by Kinard et al. [13] that about half of the isolated cells do burst, but showing shorter active phases of a few seconds, while Jonkers et al. [14] observed isolated cells with active phases of several minutes. As a different explanation, the heterogeneity hypothesis focuses on the different electrical properties of the cells and the existence of a narrow parameter window for bursting as the key reasons to understand the phenomenon [8,15-17]. A generic mechanism for the production of global oscillations called diversity is analyzed in Refs. $[18,19]$. The introduction of diversity amongst the elements destabilizes the quiescent state of an excitable medium and leads to global oscillations even when each individual element of the medium is quiescent in isolation. It is also important to mention the works by Loewenstein et al. [20,21], where a dynamic mechanism is presented by which the electrical coupling of identical nonoscillating cells can generate synchronous membrane potential oscillations. Finally, a hypothesis based on paracrine effects on the $\beta$ cells by glucagon secreted by $\alpha$ cells in the islet has been recently developed [22].

Since the pioneering work of Atwater et al. [23], in which the first biophysical model for the bursting activity in pancreatic $\beta$ cells was presented, many mathematical models have been proposed to study the electrical activity of these cells. The first model, due to Chay and Keizer [24], was based on the Hodgkin-Huxley equations for the squid giant axon [25]. During the last two decades, dozens of models have been analyzed, each of them trying to understand particular processes related to this phenomenon and making use of the most recent experimental results. Of particular importance is the appearance of simple two-dimensional (2D) 
maps that produce bursting behavior similar to the electrical activity observed in biological neurons and endocrine cells. A thorough analysis of the synchronization of these maps is developed in Refs. [26-28].

In the present paper we study a modification of the mathematical model for the pancreatic $\beta$ cell proposed by Sherman et al. [11]. We have focused our attention on the transition from the deterministic model, which represents bursting cells coupled in intact islet of Langerhans, to the stochastic model representing an isolated spiking cell. We have studied this model as a paradigmatic example of electrophysiologically bursting cell models, as we are interested in developing an approach to obtain as much information as possible from a biological system in a noisy environment. In the literature several tools have been suggested for studying irregular biological phenomena, such as heart rate variability [29] or neural spiking signals [30], both in noiseless and in noisy environments. We believe, however, that $\beta$-cell models warrant a thorough study all by themselves, and our work points in this direction. Our first goal is to examine how the dynamical properties of a bursting signal are distorted by increasing noise, in order to find the most effective tools to obtain information from such noisy signals. We have used several methods. Some are already known in the literature, but have not been applied in this context. Other methods are new, such as the analysis of the time series obtained from the number of spikes in each burst. Second, we are interested in studying the transition between the bursting and spiking behaviors of the Sherman model, as it relates two easily measurable quantities, the membrane potential and the number of cells in the cluster. Obtaining useful ways to quantify this transition might be of interest to actual biological research as the transition may influence the secretion of insulin [31,32].

We have organized the paper as follows. We start by reviewing the main features of the model, both in the deterministic and the stochastic regime. In Sec. III we study the Lyapunov exponents of the system and how they evolve with the number of cells. In Sec. IV we propose the interspike interval time series as a fruitful tool to obtain information about the periodic or chaotic nature of the system, as well as its bursting or spiking behavior, even when the level of noise is acute. In Sec. V, we analyze the time series obtained from the number of spikes in each burst, in particular the variation of their mean and standard deviation in the transition from the intact islet to the isolated spiking cell.

\section{DESCRIPTION OF THE MODEL}

The model that we have used for our investigations was originally developed by Sherman et al. [11], as an update of earlier models by Chay and Keizer [24] incorporating the experimental results of Rorsman and Trube [10]. It was formulated to examine if the hypothesis of channel sharing could explain the different electrical behavior observed in isolated and coupled cells. This model is often referred to as the supercell model, because the electrical coupling between the cells is so strong (the conductivity $g_{c}=\infty$ ) that all cells behave in total synchrony, and a cluster of cells can be considered as a single cell with the total volume of the cluster.
The Sherman model is composed of three differential equations, the independent variables being the membrane potential $V$, the concentration $\mathrm{Ca}$ of free intracellular $\mathrm{Ca}^{2+}$, and the fraction $n$ of open voltage-gated $\mathrm{K}^{+}$channels. The mathematical expression of the model is presented in the Appendix. As is usual in this kind of cellular model, $V$ and $n$ are responsible for the fast spiking process, while the equation involving $\left[\mathrm{Ca}^{2+}\right]_{i}$ describes the slow processes that regulate the transition between the active and the silent phases. This model only takes into account the ionic currents of the voltage-gated $\mathrm{K}^{+}$channels, the voltage-gated $\mathrm{Ca}^{2+}$ channels, and the calcium-activated $\mathrm{K}^{+}$channels. The latter typically shows just five or six open channels out of the several hundreds that each cell has, and the stochastic opening and closing of them is supposed to be the key to understanding the destruction of bursts in isolated cells and small clusters. A very similar model was presented by Chay and Kang [12] and the same results were obtained.

\section{A. The deterministic regime}

The model presents two regimes, the deterministic and the stochastic. We first focus our attention in the deterministic regime. It represents the electrical behavior of a cell in an intact islet of Langerhans. It does not depend on the number of cells of the cluster, as it can be seen as the limit of infinitely many cells. Depending on the parameters, we obtain a very wide variety of behaviors, such as long and short bursts, continuous spiking, bursts with no spikes, etc. We are interested in the transition from bursts to isolated spikes. Varying the $\mathrm{Ca}^{2+}$ reversal potential $V_{\mathrm{Ca}}$ proved to be a good way to reach different behavioral regimes, and hence we chose $V_{\mathrm{Ca}}$ as the active parameter. This is illustrated in Fig. 1, where the bifurcation diagram of the system is shown when $V_{\mathrm{Ca}}$ is varied. First, we note that if we vary the parameter the trajectories produce bursts of any number of spikes from 1 to 45 , the transition from one case to another occurring via a complex mixture of different bifurcations. For $V_{\mathrm{Ca}}>136.5 \mathrm{mV}$ we have regular periodic spiking, that undergoes a perioddoubling cascade to chaos when $V_{\mathrm{Ca}}$ decreases. For $V_{\mathrm{Ca}}>134.5 \mathrm{mV}$ several periodic windows of increasing period are present, starting in their right sides with saddle-node bifurcations and ending to the left in period-doubling cascades [see Fig. 1(b)]. If $V_{\mathrm{Ca}}$ is decreased even further, the transition from $i$-periodic orbits to $(i+1)$-periodic orbits finally becomes a period-adding transition, in which there is a brief coexistence of both orbits and there are no more chaotic regimes separating both behaviors. Detailed studies of this phenomenon in similar $\beta$ cell models were presented in Refs. $[33,34]$. This wide variety of behaviors will be very useful to examine the different tools that will be developed later in the paper.

\section{B. The stochastic regime}

Now that we have presented the broad range of signals that the deterministic model offers, it is time to introduce the main features of the stochastic regime. While the deterministic model represents an intact islet and could be understood as a cluster of infinitely many cells, the stochastic regime is 


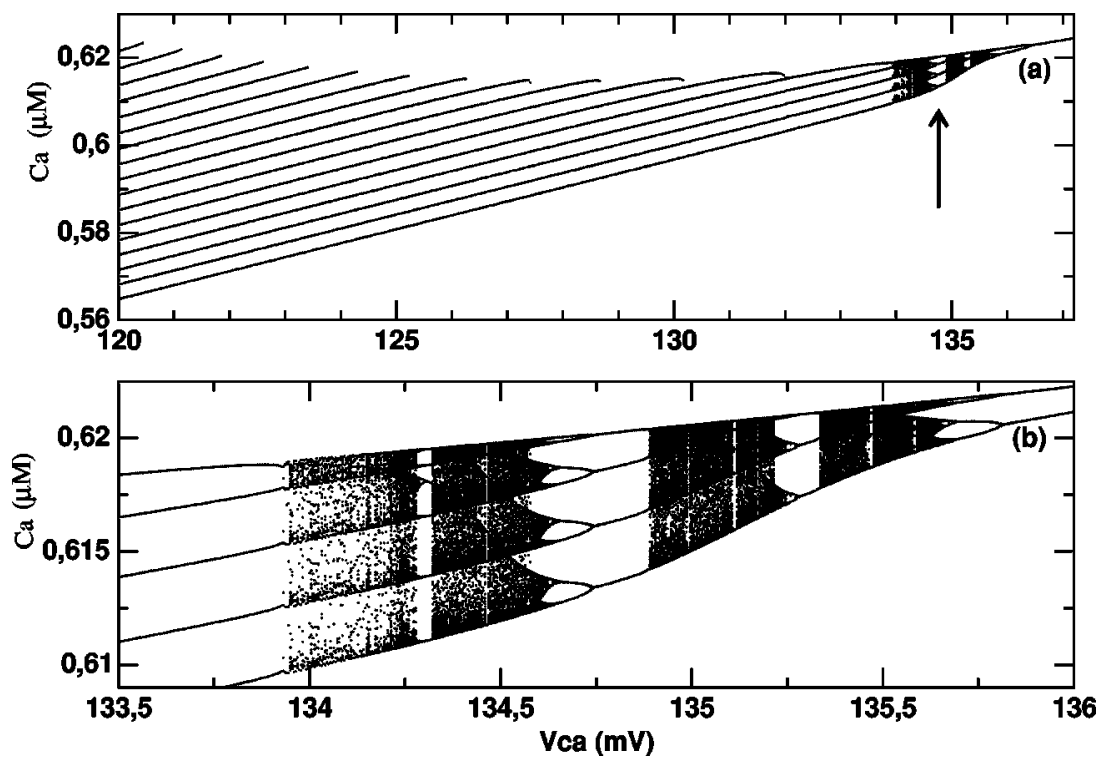

FIG. 1. (a) Bifurcation diagram of the deterministic system when the parameter $V_{\mathrm{Ca}}$ is varied. Periodic and chaotic behaviors are shown. (b) Zoom in of (a) in the region of low number of spikes per burst, marked with an arrow in (a).

governed by $N$, the number of cells in the cluster. The main idea is to substitute the expression $\mathrm{Ca} / \mathrm{Ca}+\mathrm{K}_{d}$, which represents the fraction of open channels in the deterministic regime, by the stochastic variable $p=n_{o} / n_{o}+n_{c}$ (see Appendix). $\mathrm{K}_{d}$ is the ratio between the closing and opening probability of the kinetic equation associated to the process (i.e., the calcium dissociation constant), while $n_{o}$ and $n_{c}$ are the number of open and closed calcium-activated $\mathrm{K}^{+}$channels. The variables $n_{o}$ and $n_{c}$ are stochastic and their evolution is determined by a probabilistic Poisson distribution (see Ref. [11] for more details). This substitution is valid, as the mean value of the fraction of open channels $\langle p\rangle=\mathrm{Ca} / \mathrm{Ca}$ $+\mathrm{K}_{d}$. For $\Delta t$ sufficiently small, the probability that one open channel closes in the interval $(t, t+\Delta t)$ can be approximated by

$$
P(O \rightarrow C)=\frac{n_{o} \Delta t}{\tau_{o}},
$$

while the probability that one closed channel opens in the interval $(t, t+\Delta t)$ is

$$
P(C \rightarrow O)=\frac{n_{c} \Delta t}{\tau_{c}} .
$$

$\tau_{o}$ and $\tau_{c}$ are the mean open and closed times for each channel, respectively, being

$$
\begin{gathered}
\tau_{o}=\frac{\tau_{c} \mathrm{Ca}}{\mathrm{K}_{d}}, \\
\tau_{c}=1000 \mathrm{~ms} .
\end{gathered}
$$

Sherman et al. [11] solved the stochastic system choosing the time step $\Delta t$ short enough to make the probability of opening or closing a channel in the interval $(t, t+\Delta t)$ smaller than 0.1 , ensuring then that the opening or closing of two channels in that interval of time is a rather improbable event. In this way, the fraction of open channels suffers permanent noisy perturbations after every time step, and can be under- stood as a so-called birth-and-death process [35]. While the influence of these fluctuations in the evolution of the signal is very weak if the number of channels (and also the number of cells in the cluster) is high, it will become very strong when this number is low. In fact, the stochastic signals tend to those obtained by the deterministic regime when $N$ becomes very large.

This approach for the stochastic regime has a disadvantage when considering it from a computational point of view. The time step $\Delta t \propto 1 / N$ and the calculations can therefore become extremely long when studying big clusters in order to get close to the deterministic limit. To avoid this problem and make the time step independent of the number of cells in the cluster, we make use of the central limit theorem and suppose that the result of $n$ events of probability $P$ will follow a Gaussian distribution of mean $\mu$ and standard deviation $\sigma$ expressed by

$$
\begin{gathered}
\mu=n P, \\
\sigma=\sqrt{n P(1-P)},
\end{gathered}
$$

as far as $P<0.5$ and $n P>5$. We have seen that this approximation can be applied to our case for $N>150$, while for $N<150$ we have been using the algorithm explained before. As it will be seen during the text, the matching of both algorithms around $N \approx 200$ cells is good enough from a qualitative point of view, although several quantitative results differed slightly depending on the model used. Furthermore, when $n$ becomes large, we can approximate the distribution of the probability $P$ of each event with a Gaussian of mean $\langle P\rangle$ and variance $\langle P\rangle(1-\langle P\rangle) / n$. As the standard deviation of the distribution verifies

$$
\sigma \propto \frac{1}{\sqrt{n}} \propto \frac{1}{\sqrt{N}},
$$

when the number of cells $N \rightarrow \infty, P$ tends to its mean value $\langle P\rangle$ and the stochastic model resembles the deterministic 
P6
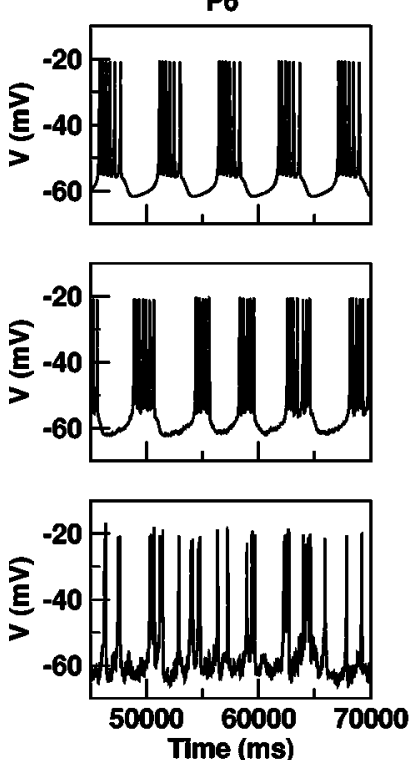

Ch
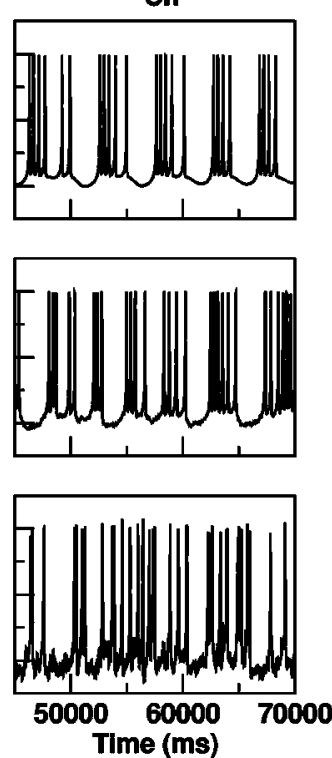

P40

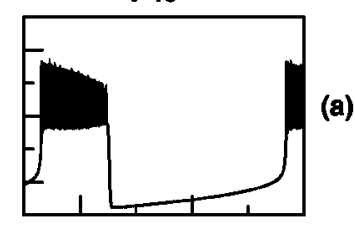

(b)
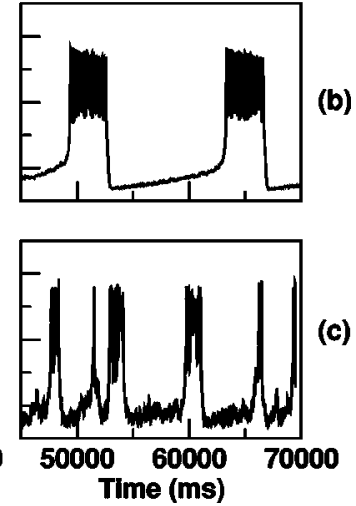

(a)
FIG. 2. Plots of the membrane potential for $P 6, C h$, and $P 40$. (a) Deterministic case, (b) $N=1000$, (c) $N=10$. We can see that the noise affects each orbit in a different way. For small values of $N$, only $P 40$ still shows a recognizable bursting behavior. model (see Ref. [12] for more details). Finally, it is worth saying that it is obviously possible to bridge the Poisson and the Gaussian approaches by considering multievent processes in the Poisson distribution.

In Fig. 2 we can see a plot for the membrane potential of a periodic orbit of 6 spikes per burst $\left(V_{\mathrm{Ca}}=131 \mathrm{mV}\right)$, a chaotic orbit $\left(V_{\mathrm{Ca}}=134 \mathrm{mV}\right)$, and a periodic orbit of 40 spikes per burst $\left(V_{\mathrm{Ca}}=111 \mathrm{mV}\right)$. From now on, we will refer to these three orbits as $P 6, C h$, and $P 40$, respectively. They have been plotted for the deterministic model, as well as for $N=1000$ cells and for $N=10$ cells in the stochastic regime. It is clear that the noise affects each orbit in a different way. While decreasing the number of cells makes the average time period of all orbits decrease, we can see that the orbits with many spikes per burst are more robust than those with few spikes per burst. For $N=1000$ it seems that the chaotic orbit still maintains its main properties and can easily be distinguished from the periodic orbits. However, for $N=10$ only the orbit of period 40 still shows structures that resemble the bursts. For such a small cluster, the two other modes already show exclusively a randomly spiky behavior. Obviously, all signals will be even more spiky if we plotted them for $N$ $=1$.

\section{LYAPUNOV EXPONENTS}

The Lyapunov exponents $(\mathrm{LE}) \lambda_{i}(i=1, \ldots, d, d$ being the dimension of the system) give useful information about the nature of the systems. Our model has three Lyapunov exponents, one of them always being equal to zero (corresponding to the direction of the flow). The other two can be either positive or negative. If at least one of the Lyapunov exponents is positive, then the orbit is considered to be chaotic, while the orbit will be periodic if all of them are smaller or equal to zero. As we showed in the last section, varying a parameter (in our case $V_{\mathrm{Ca}}$ ) we can obtain a wide range of periodic orbits, from a few to many spikes per burst, as well as chaotic orbits.
Figure 3 shows the largest $\mathrm{LE} \neq 0\left(\lambda_{L}\right.$ from now on $)$ as a function of the parameter $V_{\mathrm{Ca}}$, for different sizes of the cell cluster. The lower line corresponds to the deterministic model, that is, the limit of infinitely many cells. We can observe that most of the chaotic orbits fall in a narrow range of values of $V_{\mathrm{Ca}}$ around $V_{\mathrm{Ca}}=135 \mathrm{mV}$, that is, in the zone of small number of spikes per burst (NSB from now on). Furthermore, it is clear that $\lambda_{L}$ decreases almost linearly when $V_{\mathrm{Ca}}$ decreases, that is, when NSB increases. The calculation of $\lambda_{L}$ confirms that bursts with many spikes are more robust than those with few spikes. The other three curves in Fig. 3 were calculated for $N=5000, N=1000$, and $N=150$ cells in the stochastic regime. The values of $\lambda_{L}$ suffer a translation towards higher values when $N$ decreases. This makes sense, as the value of the largest Lyapunov exponent for a random time series should be infinity. However, even for $N=150$ cells, when the level of noise is quite high, the main features of the deterministic curve are still recognizable, and there is a range of orbits that still show negative values of the Lyapunov exponent. Furthermore, the slope of the approximately linear curve is maintained even for very small clusters. This means that even in very noisy environments we can find traces of the original properties of the orbits, their robustness, their periodic or chaotic nature, etc., and the development of tools to pursue this idea will become the main goal of our work.

Figure 4 shows the variation of $\lambda_{L}$ for the periodic orbits $P 6$ and $P 40$ and for the chaotic orbit $C h$, when we decrease the number of cells in the cluster $N$ (from right to left). We have started with a very high value of $N$, where the stochastic regime is almost indistinguishable from the deterministic regime. The values of $\lambda_{L}$ are nearly constant until a threshold value around $N=10^{6}$ is crossed. After this point, all curves start to grow and the periodic orbits eventually cross the horizontal axis. Obviously, when a periodic orbit shows a positive value of $\lambda_{L}$, it is impossible to recognize its periodic nature any more. However, as observed in Fig. 3, the value of $N$ for which this happens depends strongly on $V_{\mathrm{Ca}}$.

The Lyapunov exponents shown in Figs. 3 and 4 have been calculated making use of the algorithm proposed by 


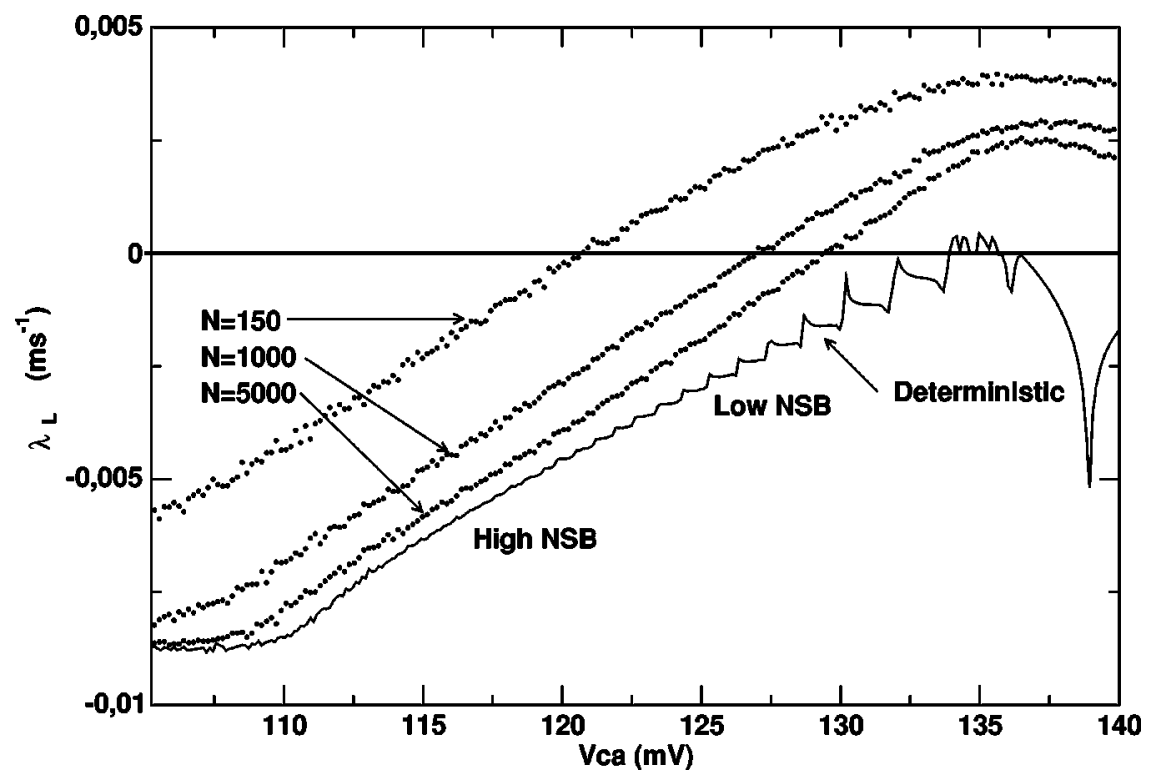

FIG. 3. Largest Lyapunov exponent as a function of the parameter $V_{\mathrm{Ca}}$ for the deterministic model, for $N=5000, N=1000$, and $N=150$ cells.

Wolf et al. [36] for mathematical models. However, we are also interested in calculating the Lyapunov exponents analyzing the signals as time series. We have used the algorithm due to Rosenstein et al. [37], already compiled in the TISEAN package [38]. In order to apply this method, it is necessary to calculate the optimum time delay for each time series, and we have chosen the value that minimizes the mutual information function. The results of $\lambda_{L}$ analyzing the signals as time series coincided with the ones obtained with the Wolf algorithm for models, both for the deterministic case and the stochastic case with high values of $N$. For very noisy signals (that is, for low values of $N$ ) extracting Lyapunov exponents from a time series is always a difficult task, but we were able to reproduce the main features shown in Fig. 3. For very low values of $N$, signals with low NSB show positive $\lambda_{L}$ and therefore are indistinguishable from chaotic orbits, while the periodicity of signals with high NSB is recognizable even in the presence of a quite high level of noise. In the following sections, we will examine in depth the differences between periodic orbits of low NSB, periodic orbits of high NSB, and chaotic orbits in noisy environments.

\section{STUDYING THE INTERSPIKE TIME INTERVALS}

Since the work of Sauer [39], in which the possibility of reconstructing chaotic attractors from interspike intervals was presented, several works have focused on the study of time series for interspike intervals [40-42]. In fact, the interspike intervals (ISI from now on) have proved to be a fundamental source of information to characterize the nature of many biological signals. Furthermore, they are easily measurable in experimental data, and this fact encouraged us to study them in our system.

\section{A. ISI return maps}

The study of return maps is a well known method to distinguish chaotic from periodic signals in systems with weak

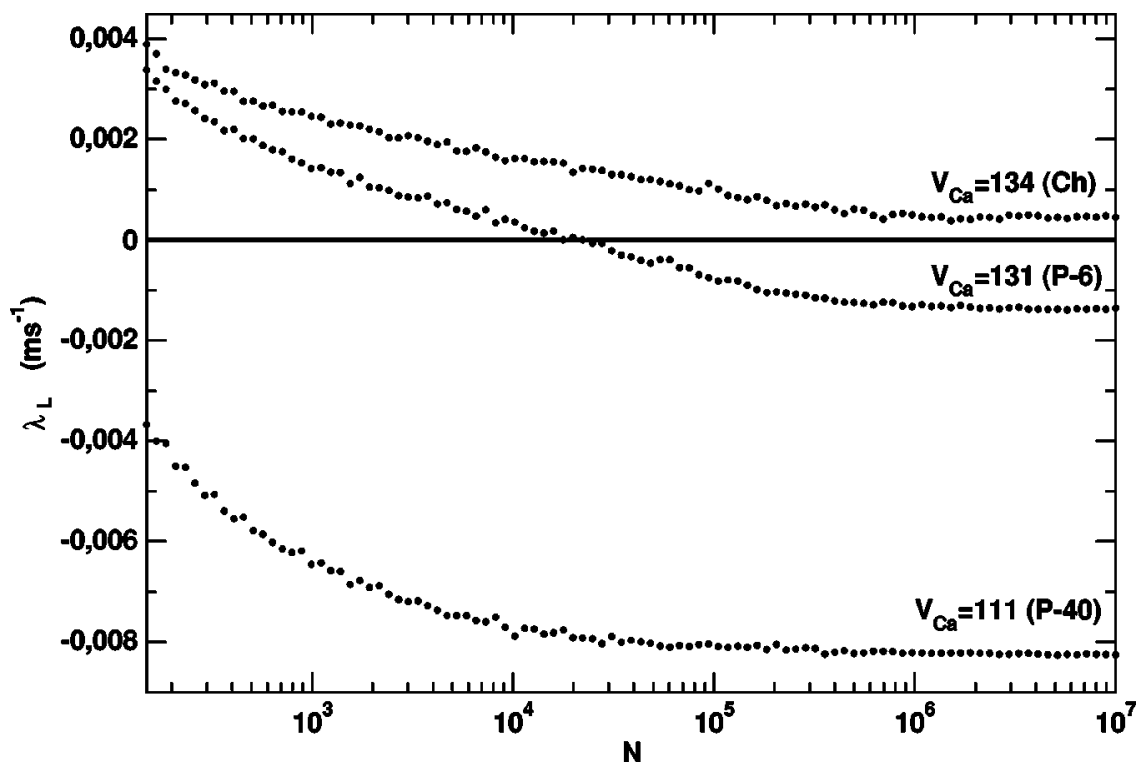

FIG. 4. Largest Lyapunov exponent as a function of $N$ for the periodic orbits $P 6$ and $P 40$, as well as for the chaotic orbit $C h$. 

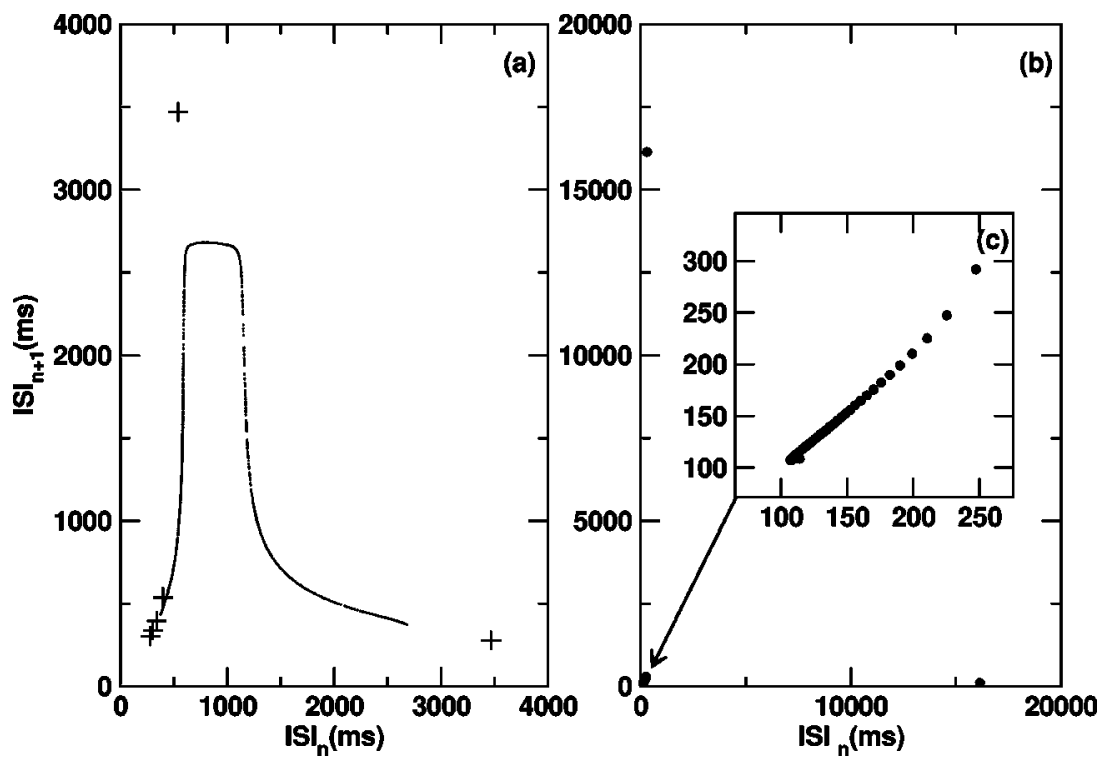

FIG. 5. (a) ISI return maps for $P 6$ (six crosses) and the chaotic orbit (dots). (b) ISI return map for $P 40$. (c) Zoom in of the region marked with an arrow in (b). The return maps for the periodic orbits are composed of three isolated groups of dots, while the chaotic signal gives rise to an apparently continuous curve.

chaos. We have started our approach to ISI analysis studying qualitatively the return maps of the ISI time series, in which we plot the $(n+1)$ th ISI against the $n$th ISI. The six small crosses in Fig. 5(a) show the return map for the periodic orbit P6 $\left(V_{\mathrm{Ca}}=131 \mathrm{mV}\right)$, while the continuous curve in the same figure shows the return map for $C h\left(V_{\mathrm{Ca}}=134 \mathrm{mV}\right)$. Figure 5(b) shows the return map for the periodic orbit $P 40$ $\left(V_{\mathrm{Ca}}=111 \mathrm{mV}\right)$ and in the inset a zoom of this picture is plotted. It is remarkable that the return maps for the periodic orbits are composed of three isolated groups of dots, while the chaotic signal gives rise to an apparently continuous curve. Strictly speaking this curve is not one dimensional, as a chaotic attractor necessarily shows a fractal dimension [43]. Due to the low chaoticity of this model, a unimodal map like this one would reproduce very precisely the ISI time series of our 3D continuous time model. Similar results have been obtained, for example,for the BelousovZhabotinskii reaction [44,45], among many other systems. Furthermore, this kind of map gives rise to an intermittency phenomenon. In our case, the trajectory spends the active phase of the bursting activity developing several iterations close to the bottom-left extreme of the map, and eventually escapes from it to make a big loop that represents the silent phase.

The apparent continuity of the chaotic curve shows that almost all ISIs between a minimum and a maximum value are possible, while there is a forbidden range in the periodic case. This qualitative difference has proved to be of significant importance when trying to characterize the nature of an orbit in a noisy environment. Figure 6 shows the return maps for the same three orbits $(P 6, P 40$, and $C h)$ for $N=5000$ and $N=500$ cells. We can see that for a certain amount of noise $(N=5000)$, the periodic orbit still shows three isolated groups of dots and, therefore, its periodic nature is clearly recognizable. The dots with small ISI $(n)$ and ISI $(n+1)$ represent interspike intervals between two spikes in the same burst, while the dots with one high coordinate represent the long interspike interval between two different bursts. As

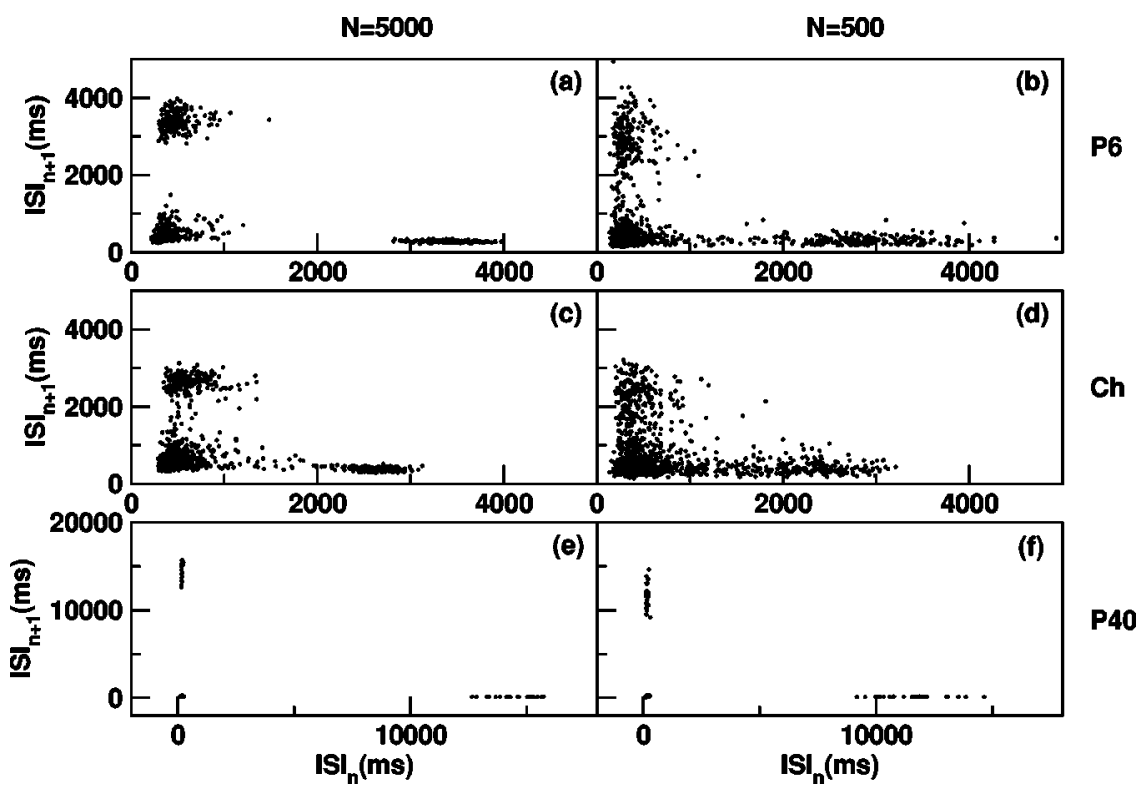

FIG. 6. ISI return maps for $P 6$, $C h$, and $P 40$. The pictures to the left correspond to $N=5000$ cells, while the pictures to the right correspond to $N=500$ cells. The three groups of dots in the return map of a periodic orbit become more and more spread when the size of the cluster decreases (and therefore when the noise is increased). 

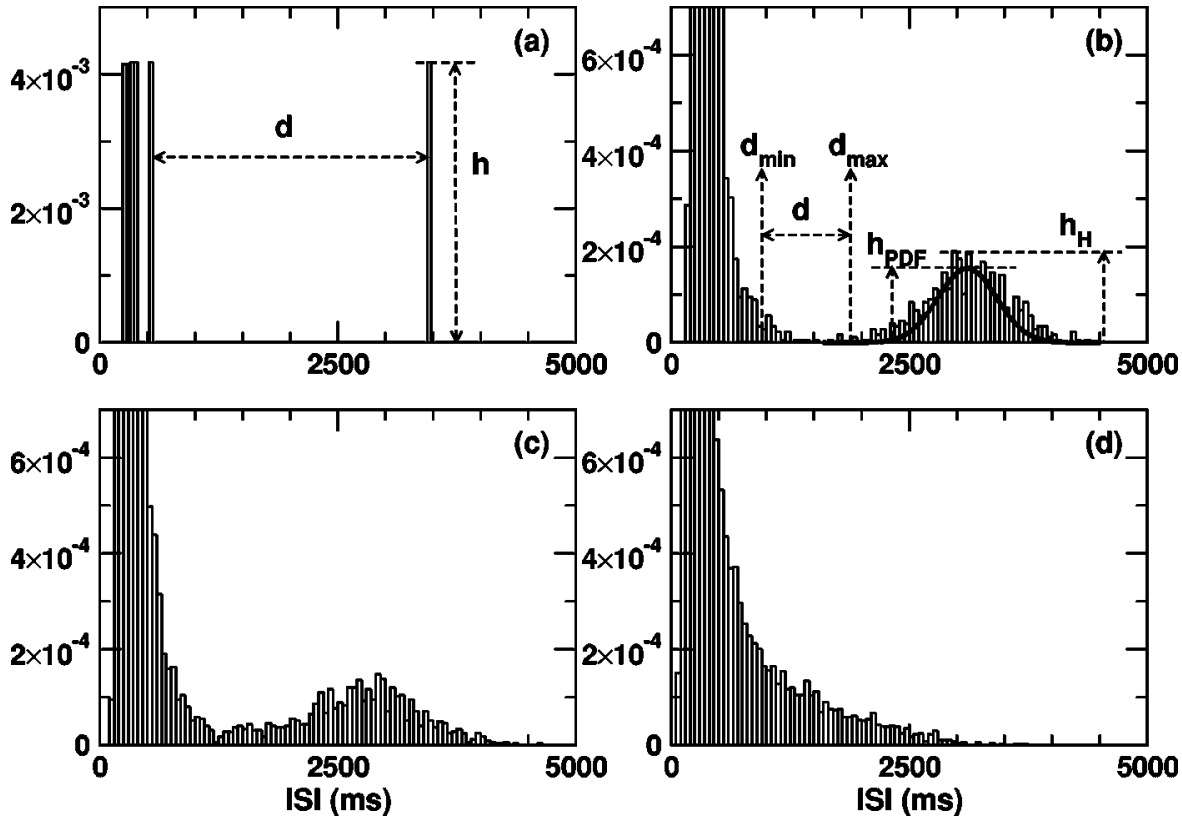

these three groups of dots get more and more spread because of the increasing value of the noise, the distance between them diminishes. Eventually there is a value of the number of cells $N_{d}$ that makes all points collide in an indivisible single group. Using this method, a periodic orbit is indistinguishable from a chaotic one for $N<N_{d}$, but we will be able to characterize its periodic or chaotic nature if $N$ is higher than this critical value. $N_{d}$ depends on each orbit and a robust method to measure it must be defined. If we examine Fig. 6(b) with the naked eye, we can deduce that for $N=500, P 6$ is already mixed, and therefore it shows a similar aspect to a chaotic orbit with the same amount of noise [see Fig. 6(d)]. This means that, at least as a rough guess, $N_{d} \geqslant 500$ for $P 6$, while for that value of $N, P 40$ clearly maintains its periodic nature and still shows a gap between the three different groups of dots [see Fig. 6(e) and 6(f)]. However, the use of numerical techniques related to clustering theory can increase substantially the effectiveness of this method, obtaining lower values for $N_{d}$, and distinguishing periodic from chaotic signals even in very noisy environments. This fact will be shown in the following section.

\section{B. ISI histograms}

In order to develop these ideas and obtain more quantitative results, we have applied several numerical techniques to the histograms of interspike intervals. The ISI histogram is a very powerful tool, because it gives information about both the periodic or chaotic nature of the orbit and about its bursting or spiking character.

\section{The periodic or chaotic nature of the orbit.}

If an orbit is periodic, then the histogram consists of several disconnected bars, each of them representing one ISI (assuming that the bars are thin enough). This is seen in Fig. 7(a) for $P 6$. If the bursts have $n$ spikes, then $n-1$ bars will have low values of ISI and will represent the interspike time interval due to spikes belonging to a certain burst. However, one bar, representing the interval between the first spike of a burst and the last spike of the previous burst, will be found at high values of the interspike time interval. In the presence of small noise, the first group of $n-1$ bars will become a continuous distribution, and the isolated bar will be widened into a Gaussian-like curve. We have named $d$ the distance between the closest extremes of both peaks and this quantity will be very useful for our analysis $\left(h_{H}\right.$ and $h_{P D F}$ will be defined in the following section).

In a chaotic orbit, even in the absence of noise the ISI histogram follows a continuous curve, made out of several peaks [see Fig. 8(a)]. Therefore, the value of $d$ is zero for a chaotic orbit no matter how noisy the system is, but for a periodic orbit $d$ will decrease and tend to zero when the noise of the system grows and makes the two peaks come closer. From this point of view, $d$ becomes a natural parameter to distinguish a chaotic orbit from a periodic orbit in a noisy environment.

Figure 7 shows the ISI histogram for the periodic orbit $P 6$ and different values of $N$, while Fig. 8 shows the same curves for the chaotic orbit $C h$. The height of the second peak, that we will call $h_{H}$, and the distance between peaks $d$ are shown. Figures 7(a) and 8(a) show the deterministic case for each orbit. Figures 7(b) and 8(b) show the histograms for $N=1000$. If the noise grows strongly, as it is shown in Fig. 7 (c) and $8(\mathrm{c})$, where $N=500$, then the tails of both peaks come in contact in the periodic case and we can no longer distinguish a periodic orbit from a chaotic one (unless we make use of numerical clustering techniques). Finally, Figs. $7(d)$ and $8(d)$ show the histograms for $N=100$, which are the typical ones expected for a random spiking process.

As was already mentioned, measuring $d$ by analyzing the histograms with the naked eye can result in rather ineffective measurements, especially for low values of $N$. First, it might be difficult to separate the dots that belong to each peak in very noisy signals. To solve this problem, we have made use of the Hartigan's k-means clustering algorithm [46,47]. This 

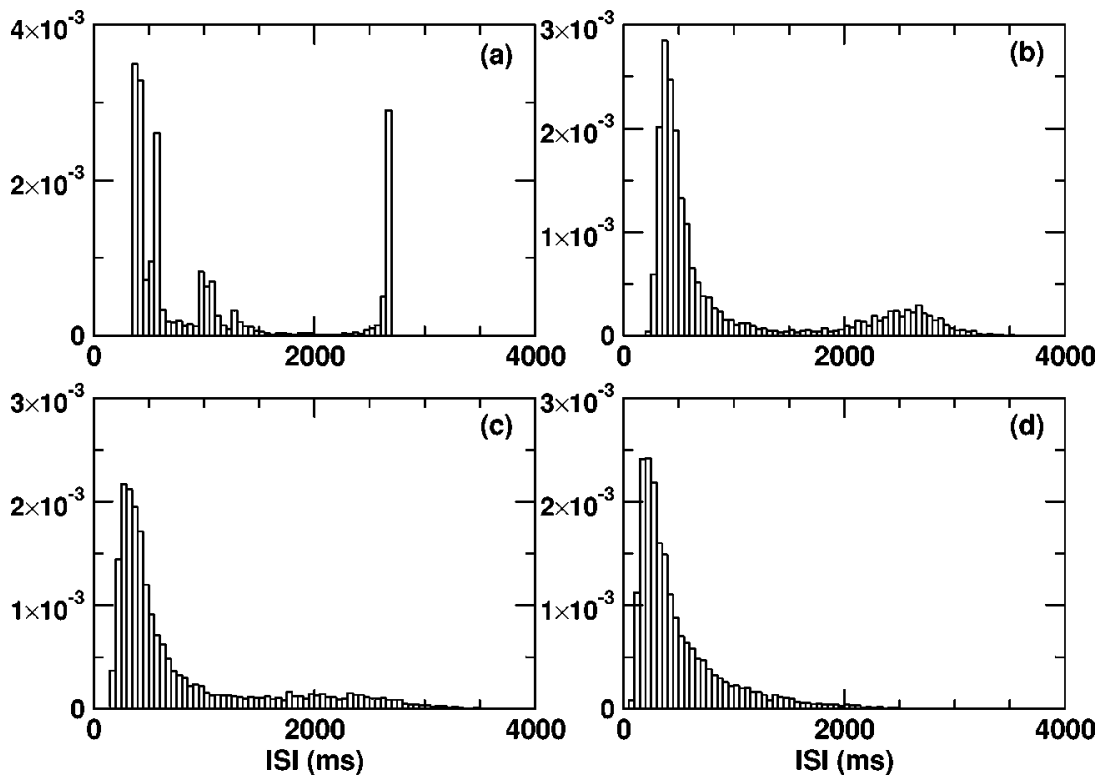

FIG. 8. ISI histograms for the chaotic orbit $C h$. (a) Deterministic case, (b) $N=1000$, (c) $N=500$, (d) $N=100$. In a chaotic orbit, even in the absence of noise the ISI histogram follows an apparently continuous curve.

numerical technique classifies the dots into two clusters, the ones that represent interspike intervals and the ones that represent interburst intervals. Second, we must obtain robust values of $d$ that are not strongly influenced by isolated measurements or the length of the time series. A good method is to suppress the spurious isolated values placed in the gap between both peaks erasing the highest $1 \%$ of the cluster to the left and the lowest $1 \%$ of the cluster to the right. After these two transformations, we measure $d_{\min }$ as the maximum value for the remaining cluster on the left, $d_{\max }$ as the minimum value for the remaining cluster on the right, and $d$ $=d_{\max }-d_{\min }$ as the distance between both values [see Fig. 7(b) for an example].

In Fig. 9 we have plotted the variation with $N$ of $d_{\text {min }}$, $d_{\text {max }}$, and $d$, for the periodic case $P 6$, when we apply the clustering algorithm. We have also plotted the values of $d$ that were obtained with the naked eye to compare the effi- ciencies of both methods. We are interested in measuring $N_{d}$, that is, the value of the number of cells that makes all points collide in a single inseparable group, and, therefore, makes it impossible to distinguish the periodic orbit from a chaotic one when $N<N_{d}$. As we already said, a chaotic orbit should show $d=0 \mathrm{~ms}$ for all values of $N$. However, as our method erases several measurements from a region of very low population, a finite gap appears also in chaotic time series. Our algorithm has proved to give values of around $d_{C h}$ $\approx 200 \mathrm{~ms}$ for all values of $N$ in $C h$. Therefore, a periodic orbit should show sufficiently bigger gaps to be recognizable. As a criterion, we have decided to define $N_{d}$ for a periodic orbit as the value of $N$ for which $d=d_{0} \approx 1.5 d_{C h}$ $\approx 300 \mathrm{~ms}$. Following this criterion, and observing Fig. 9, $N_{d} \approx 300$ cells for $P 6$. The high efficiency of this method is remarkable, as the level of noise that the signal suffers when $N=300$ cells is quite high. We measured $d$ directly from the

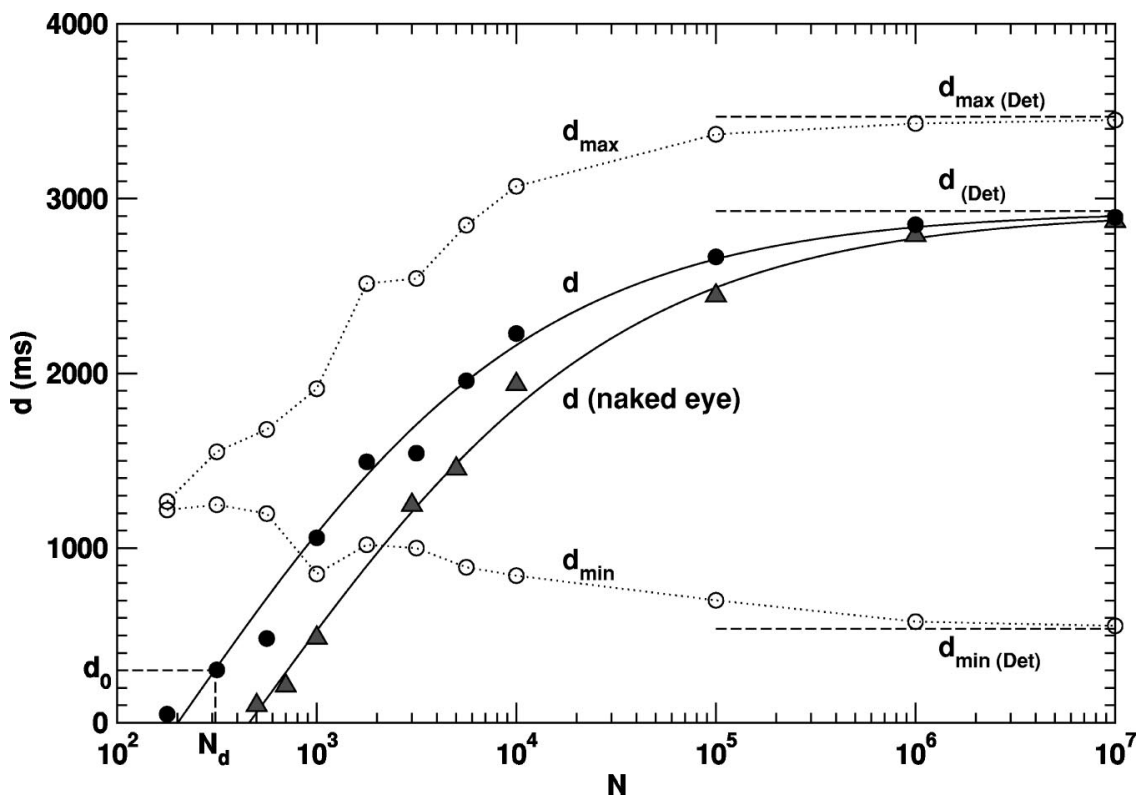

FIG. 9. Variation with $N$ of $d$, $d_{\text {max }}$, and $d_{\text {min }}$, for $P 6$, when we apply the Hartigan's clustering algorithm (circles). The values of $d$ obtained from direct measurement are also shown (triangles). $N_{d}$ $\approx 300$ for $P 6$. The two solid lines represent mathematical approximations of $d$. 


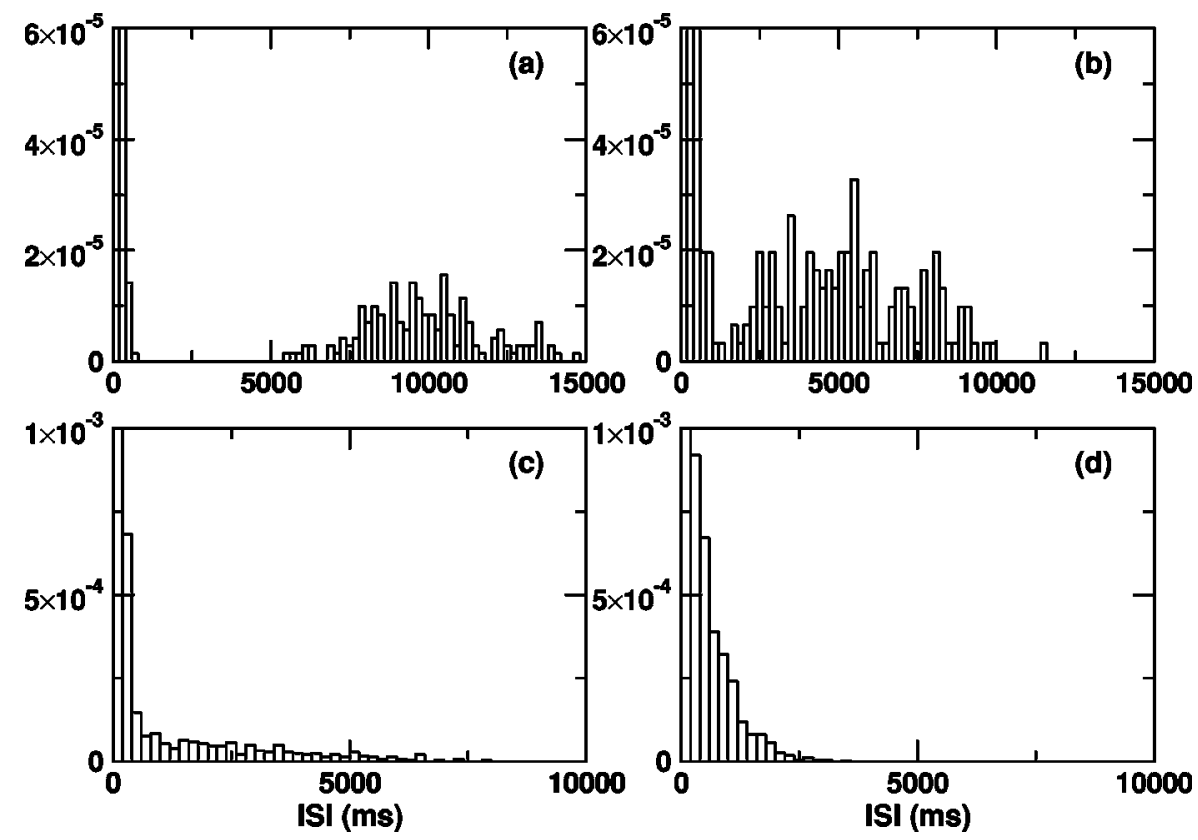

FIG. 10. ISI histograms for P40. (a) $N=150$, (b) $N=25$, (c) $N=10$, (d) $N=1$. The higher number of spikes per burst, the more robust to noise the signals are.

histograms and we obtained much higher values of $N_{d}$ (around $N_{d} \approx 500$ for $P 6$, as it was shown in the preceding section, and can be now observed in Fig. 9).

The relations between $d$ and $N$ plotted in Fig. 9 have proved to fit very accurately a mathematical expression of the form

$$
d=d_{\text {Det }}[\tanh (a \ln N+b)]=d_{\text {Det }}\left[\frac{A N^{B}-1}{A N^{B}+1}\right],
$$

where $d_{\text {Det }}=2930 \mathrm{~ms}, A=0.077 \pm 0.008, B=0.48 \pm 0.01$, for the higher curve (corresponding to the clustering algorithm), and $d_{\text {Det }}=2930 \mathrm{~ms}, A=0.057 \pm 0.005, B=0.47 \pm 0.01$, for the lower curve (corresponding to the direct measurement). The correlation coefficients are $r=0.9977$ and $r$ $=0.9984$, respectively. We can observe that the exponent $B$ of the number of cells $N$ is approximately equal to $1 / 2$. In fact, the dependence of different signal properties on $\sqrt{N}$ will be present all throughout the work and we will refer to it later in the paper.

Finally, it is reasonable to examine whether the number of spikes per burst might influence the stability of the signal. Figure 10 shows the ISI histograms for $P 40$ and four very low values of $N$. It is clear once again that the more spikes per burst (NSB), the more robust to noise the orbits are. For high values of NSB, we obtain lower $d_{\text {min }}$ because the spikes in the burst become narrow and higher $d_{\max }$ because the silent phases get longer. Hence, higher $d=d_{\max }-d_{\text {min }}$ are obtained and $N_{d}$ is smaller than for orbits with lower NSB. In the case of $P 40, d \approx 6500 \mathrm{~ms}$ when $d(P 6)=d_{0}$, that is, around $N=300$. As $N \leqslant 150$ in Fig. 10 , it was necessary to use the $N$ dependent time-step stochastic method to calculate these curves, and therefore this figure was far more time consuming than the other ones. Figure 10(a) shows the histogram for $N=150$, where it is clear that the periodicity of the signal is still recognizable. Only when we examine clusters of around 25 cells do the tails of both peaks touch each other [see Fig. 10(b)], and applying our clustering method we see that $N_{d} \approx 15$ cells for $P 40$. Figure 10 (c) shows the histogram for $N=10$ and now the second peak is no longer present. However, there is a long tail, which means that for this level of stochasticity the signal presents a mixed nature of varied bursts and isolated spikes (see Fig. 2). Finally, Fig. $10(\mathrm{~d})$ presents the typical histogram expected for a random spiking signal.

\section{The bursting-spiking transition}

Our system shows bursts for most values of the parameters. This behavior is shown in the ISI histograms as two differentiated peaks. However, if the system becomes very noisy, the organized structure becomes spiky, such that the histograms tend to show a unique peak. In order to characterize this transition, we have measured $h$, the height of the second peak. This height tends to zero when the system gets more and more noisy and, therefore, we can say that the system does not show bursts any more when the two peaks become indistinguishable in the histogram [see Figs. 7(d) and $8(\mathrm{~d})$, where $N=100]$.

The shape of a histogram is in fact an approximation of the probability density function of the signal (PDF from now on). The histogram only tends to the invariant curve when there is a large number of points for each bar and the width of the bars tends to be very narrow. Then, for finite data, the height of a peak in a typical histogram depends strongly on the width of the bars and the length of the data series. For this reason, we have measured the height $h_{H}$ directly from the histograms, and we have also calculated $h_{P D F}$ approximating the second peak with a mathematical expression. We have verified that the secondary peaks fit quite accurately to a normal distribution by applying a Gaussian quantilequantile test to the data. After calculating the average $\mu$ and the standard deviation $\sigma$ for each signal, we obtain the maximum of the Gaussian fit as 


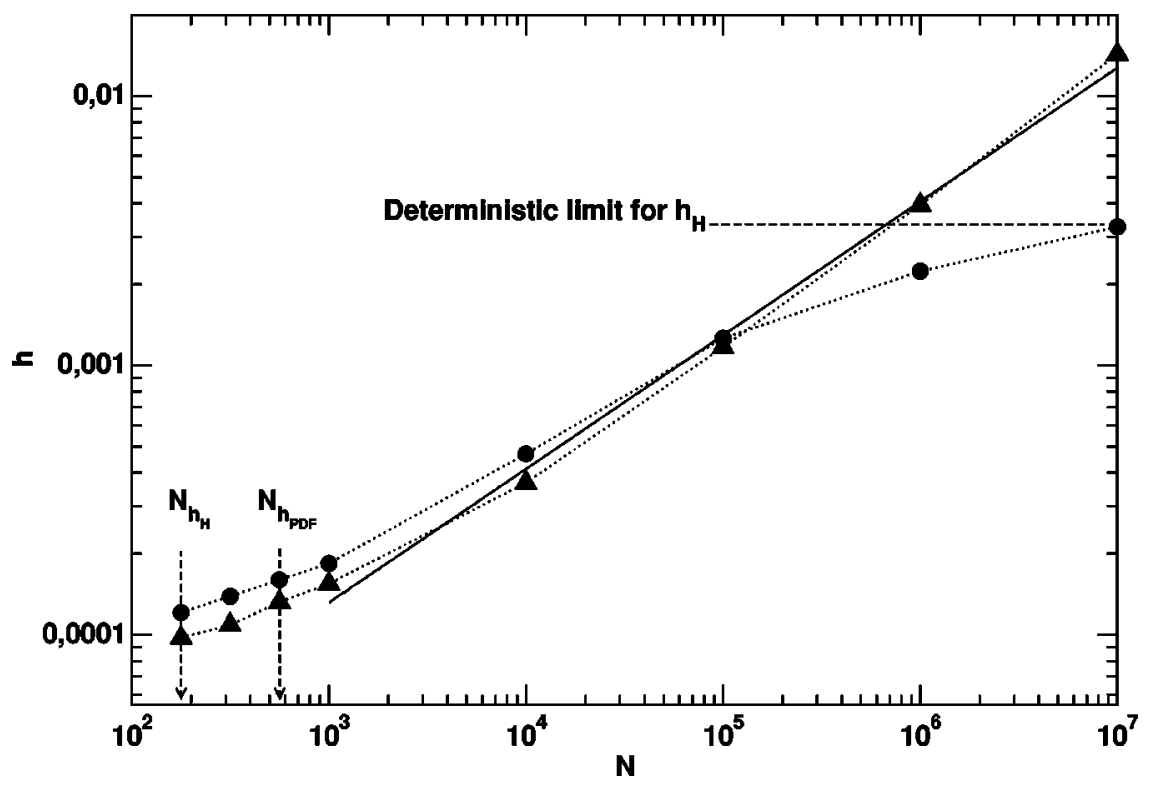

FIG. 11. Variation of $h_{H}$ (circles) and $h_{P D F}$ (triangles) for $P 6$ with the number of cells $N$. The solid line is the mathematical approximation of $h_{P D F}$.

$$
h_{P D F}=\frac{1}{\sqrt{2 \pi \sigma^{2}}}\left(\frac{n_{2}}{n_{t}}\right) .
$$

The term $n_{2} / n_{t}$, where $n_{2}$ is the number of data points in the right cluster and $n_{t}$ the total length of the ISI time series, is necessary to take into account the totality of the data in the normalization. Figure $7(\mathrm{~b})$ shows $h_{H}, h_{P D F}$, and the Gaussian approximation for the second peak of $P 6$ for $N=1000$ cells.

Figure 11 shows the variation of $h_{H}$ and $h_{P D F}$ for the periodic orbit $P 6$ when $N$ is varied. We can see that for high values of $N, h_{P D F}$ and $h_{H}$ show very different behavior. This is due to the fact that the bars of our histograms have a fixed width of $w_{H}=50 \mathrm{~ms}$, and therefore the Gaussian fit for signals with very little noise is so narrow that these bars are far too wide for them. For that value of $w_{H}, h_{H}$ tends to the fixed value of $\left(1 / w_{H}\right)\left(n_{2} / n_{t}\right)=0.003333 \ldots$. However, $h_{P D F}$ grows indefinitely, as the probability density function will tend to a Dirac delta function in the deterministic limit. As we can see in the figure, $h_{H}$ and $h_{P D F}$ are very similar for $N<10^{5}$, as for this range of $N$ the Gaussian curves are wide enough to be accurately approximated by the histograms with $w_{H}=50 \mathrm{~ms}$. However, $h_{H}$ shows slightly higher values than $h_{P D F}$. This is very common in mathematical approximations of histograms, in which the typical noisiness of the histograms makes the maximum of the fitting generally lower than the height of the highest bar [see Fig. 7(b) for an example].

If the noise is sufficiently high, the tails of the two peaks superimpose one onto the other, and the calculation of $h_{P D F}$ starts to lose precision giving exceedingly high results. We have labeled $N_{h_{P D F}}$ the approximate value beyond which the second peak becomes significantly different from a Gaussian and therefore this technique begins to fail. The curve of $h_{P D F}$ can be very accurately approximated by an expression of the type

$$
h_{P D F}=A N^{B} .
$$

If we use for the fitting the values with $N \geqslant N_{h_{P D F}}$, in order to avoid the values of $N$ for which both peaks superimpose, we obtain $h_{P D F}=4 \times 10^{-6} N^{0.50 \pm 0.02}$. The correlation coefficient of the linearization of this expression is $r=0.9975$. According to this approximation and taking into account Eq. (9), the standard deviation of the Gaussian approximation satisfies

$$
\sigma=\frac{1}{\sqrt{2 \pi h_{P D F}^{2}}}\left(\frac{n_{2}}{n_{t}}\right) \propto \frac{1}{h_{P D F}} \propto \frac{1}{\sqrt{N}} .
$$

It is worth noting its relation with the real source of the noise in our system. As discussed in Sec. II, the channel opening and closing probabilities of a single channel follow a Gaussian distribution of mean $\mu=\langle p\rangle$ and standard deviation $\sigma$ $\propto 1 / \sqrt{N}$. This fact points to the possible existence of a general law, in which many different phenomena related to this system are scaled by the square root of the number of cells $\sqrt{N}$ (as it happened, for instance, in the relation between $d$ and $N$ ).

If we decrease the size of the cluster below $N_{h_{P D F}}$, there is a value $N_{h_{H}}$ for which the second peak becomes flat $\left(N_{h_{H}}\right.$ $\approx 200$ cells for P6). For $N<N_{h_{H}}$, it is not possible to find a duration of the silent phase that is more probable than the rest, and therefore we can say that beyond this point we have a bursting-spiking coexistence, described by a unique long tail. This long tail finally disappears and for very low values of $N$ we can only distinguish a spiking behavior.

We have calculated the same curve for $C h$ and $P 40$ and very similar results were obtained for the chaotic signal. In the case of $P 40$ the slopes of the curves are also very similar to the ones shown in Fig. 11. However, this case shows much smaller values of $h_{H}$ and $h_{P D F}$ because the relative weight of the second peak in the histogram is very small; in fact it only represents one spike out of 40 . This makes, obviously, the acquisition of data a harder task.

\section{TIME SERIES OF NUMBER OF SPIKES PER BURST}

In the last section of this work, we consider the variation of the time series formed by the number of spikes per burst 


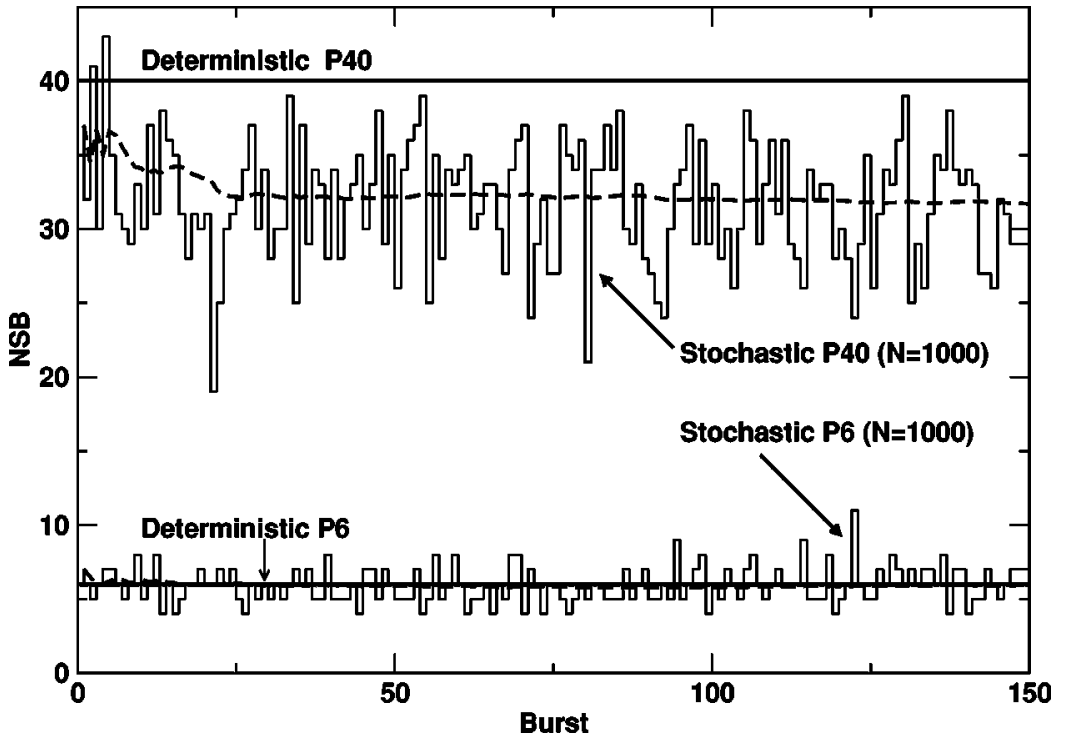

FIG. 12. Evolution for $P 6$ and $P 40$ of the number of spikes per burst with the time, during the first 150 bursts. The curves are plotted for the deterministic case (wide horizontal lines in NSB $=6$ and $\mathrm{NSB}=40$ ) and $N=1000$ in the stochastic regime. The dashed lines represent the convergence of the mean $\mu$ in both cases.
NSB, when we decrease the number of cells. As we already mentioned in the Introduction, the model of $\beta$ cell that we are studying was created to explain why the bursting behavior of intact cells in clusters is converted into random spikes in isolated cells [11]. Our target in this section is to study such transition, in particular making use of a quantity that again is easy to measure experimentally, the NSB time series. It is known that a precise study of a time series might be done from different perspectives and many different tools can be used. We have worked calculating the mean and the standard deviation of different signals.

Figure 12 shows the NSB time series for $P 6$ and $P 40$, for the deterministic regime and an example of the stochastic regime. In the deterministic regime, all bursts show the same number of spikes if the orbit is periodic. Therefore, the mean $\mu$ is an integer and the standard deviation $\sigma$ is zero. However, when noise is introduced, the NSB signals start to fluctuate making $\mu$ and $\sigma$ vary. The dashed lines in Fig. 12 plot the convergence of the mean $\mu$ for $P 40$ and $P 6$ when $N$ $=1000$.

\section{A. Evolution of NSB in the presence of noise}

We can observe that the noise makes some bursts show more spikes than the deterministic signal, while others die before reaching this value. To understand this double behavior, the system must be analyzed as the interaction between a 2D fast subsystem and the influence of a slow variable working as a parameter. As discussed by Belykh et al. [48], this approach is incomplete and, for instance, cannot explain the existence of chaotic dynamics. However, it is sufficient for our purposes. Figures 13(a) and 13(b) show the deterministic $P 6$ and $P 40$ orbit in the $V$-Ca plane. The $z$ nullcline for $V$ $-n([d V / d t]=[d n / d t]=0)$ and the nullcline for $\mathrm{Ca}(d \mathrm{Ca} / d t$ $=0)$ are plotted in both figures. The $z$ nullcline is formed by the fixed points of the fast subsystem. These fixed points are plotted with a solid line if they are stable and with a dashed line if they are unstable. The highest branch of the $z$ curve corresponds to an unstable fixed point labeled with FP1. It is surrounded by a limit cycle. The middle branch is formed by a saddle point FP2 whose manifolds surround the limit cycle and prevents the orbit from jumping down to the silent phase. The lowest branch of the $z$ curve corresponds to the stable fixed point FP3, which attracts the orbit in the silent phase. The typical dynamics of the signal is to follow the limit cycle turning around FP1, while $\mathrm{Ca}$ increases. When the limit cycle collides with the saddle point FP2 in a homoclinic bifurcation, the manifolds of FP2 collapse into a homoclinic orbit which destroys the limit cycle. In the following moment, the homoclinic orbit is destroyed, and the unstable manifold of FP1 becomes the stable manifold of FP2 forming a heteroclinic orbit [see Fig. 13(c) for a diagram of the manifolds of the fixed points in that moment]. As the
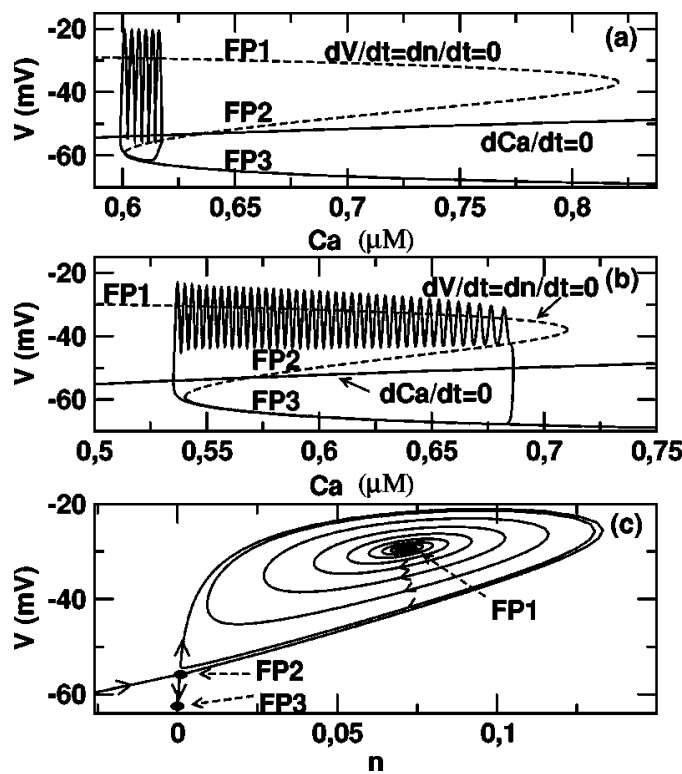

FIG. 13. (a) Deterministic signal for $P 6$ in the $V$-Ca plane. (b) Deterministic signal for $P 40$ in the $V$-Ca plane. The $V$ - $n$ and the $\mathrm{Ca}$ nullclines are plotted both in (a) and (b). (c) Unstable points FP1 and FP2, stable point FP3 and their invariant manifolds for $P 6$, just after the homoclinic bifurcation. 


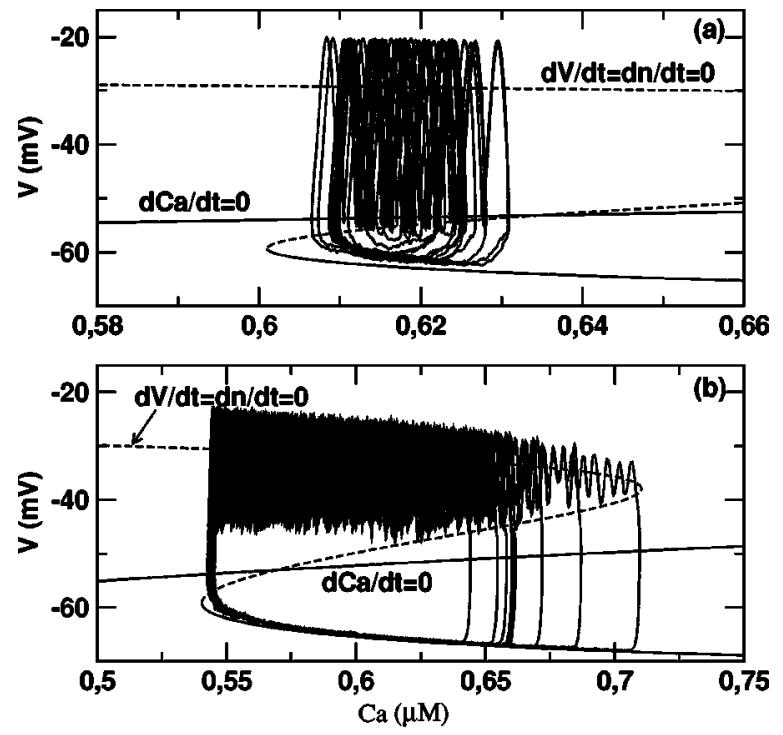

FIG. 14. Stochastic signals for $P 6$ (a) and $P 40$ (b) in the $V$-Ca plane, when $N=1000$. The $V-n$ and the Ca nullclines are plotted.

limit cycle has disappeared, the only remaining attractor is FP3. This is the end of the active phase, as the trajectory is obliged to cross the $\mathrm{Ca}$ nullcline and relaxes into the stable fixed point FP3 while Ca decreases. After some time, FP3 collides with FP2 in a saddle-node bifurcation, and the trajectory restarts the process reaching the active phase and following once again the limit cycle.

Figure 14 shows the $P 6$ and $P 40$ orbits when $N=1000$, plotted in the $V$-Ca plane. When the system suffers some kind of noise, the situation is transformed in the following manner. While the trajectory is turning around the limit cycle inthe active phase, the boundary between the limit cycle basin and the FP3 basin is formed by the stable manifolds of FP2. A particularly strong perturbation might push the particle beyond this boundary, make it cross the middle line of the $z$ nullcline, and reach FP3 and the silent phase. This mechanism explains the bursts that show fewer spikes than the deterministic orbit and it was already studied in Ref. [11]. However, we have found two different mechanisms that explain the existence of extra-long bursts in a noisy environment. To present the first one, we must analyze the invariant manifolds of the three fixed points shown in Fig. 13(c), for a value of $\mathrm{Ca}$ just beyond the one that corresponds to the homoclinic bifurcation. First, the orbit must survive in the presence of noise until it reaches the point in which the limit cycle collides with FP2 and gets destroyed. Up to that point, it has already made as many spikes as the deterministic signal. The typical behavior is that it leaves the active phase and falls to the stable fixed point FP1. However, the unstable manifold of FP1 has become the stable manifold of FP2 via a heteroclinic connection. As the unstable manifold of FP1 used to die in the limit cycle, it still shows a very dense spiral around FP1 before reaching FP2. For this reason, if the orbit is pushed by the noise towards the interior of this spiral, the trajectory can make several extra spikes before getting close to FP2 and then reaching FP3. This situation cannot be repeated indefinitely, however, and there is a maximum number of spikes per burst. When the value of $\mathrm{Ca}$ reaches the right knee of the $z$ curve, the unstable point FP1 collides with FP2 in a saddle-node bifurcation. In that moment, the heteroclinic spiral disappears and the particle is necessarily pushed to the remaining attractor FP3, no matter how strong the noise is. In fact, the maximum $\mathrm{NSB} \approx 45$ for the values of the parameters we are using. Figure 14(b), where an example of a noisy $P 40$ orbit is plotted, only shows two cases of these extra-long bursts, giving us an idea of the infrequent occurrence of this phenomenon.

The second mechanism that allows the trajectories to show longer bursts and compensate the noise-induced premature death of the active phase is only possible for systems with low NSB. We have named it the reinjection effect and can be understood if we analyze in detail the noisy $P 6$ orbit in Fig. 14(a). In that figure we can observe that the orbits for $P 6$ and $P 40$ show several evident differences. We must remember that $P 6$ is less robust than $P 40$ (the largest Lyapunov exponent $\lambda_{L}$ of $P 40$ is much smaller than the $\lambda_{L}$ of $P 6)$. For this reason, $P 6$ shows strong noisy fluctuations when crossing the middle branch of the $z$ nullcline (the dashed line) in its way to the lower branch, that is, to the silent phase. The orbit crosses the $\mathrm{Ca}$ nullcline just before crossing the $z$ nullcline, and therefore it tends to move to the left (to lower values of the calcium, Ca). As the orbits are not so robust, they start a pseudorandom walk backwards. Sometimes, this erratic movement reaches the attractor FP1 and the signal is pushed to the silent phase. However, we can see in Fig. 14(a) that for sufficiently strong noise it is quite common that the orbits cross the middle branch of the $z$ nullcline, move to the left in the region between the middle and the lower branch, and restart a new spike in the middle of the burst. This little loop in the $V$-Ca plane takes so little time that the new burst is born totally joined to the old one, resulting in an extra-long burst. Furthermore, the reinjection effect can be very frequent, because the orbit does not need the help of noise to survive up to the homoclinic bifurcation, as it happened with the former mechanism. Nevertheless, this phenomenon does not exist in systems with high NSB. We can see in Fig. 14(b) that for P40, when the orbits cross the dashed line of FP2 and go down to the attractor FP1, they follow an approximately straight line. This is because they are much more robust and do not show the erratic movement seen for P6. However, another important fact is that in this case the middle branch of the $z$ nullcline is over the $\mathrm{Ca}$ nullcline. Therefore, when the trajectories cross the dashed line the system pushes them to the right until they reach the Ca nullcline, making impossible the reinjection until they are too far away from the limit cycle.

\section{B. Dependence of the mean value of NSB on the number of cells $N$}

In order to measure quantitatively the relation between the NSB time series and the number of cells $N$, we must take into account that measuring the number of spikes in each burst is not always an easy task, as in very noisy environments it might be difficult to define the concept of burst. Several methods can be used. We have focused our interest on studying the membrane potential, as this potential is easy 

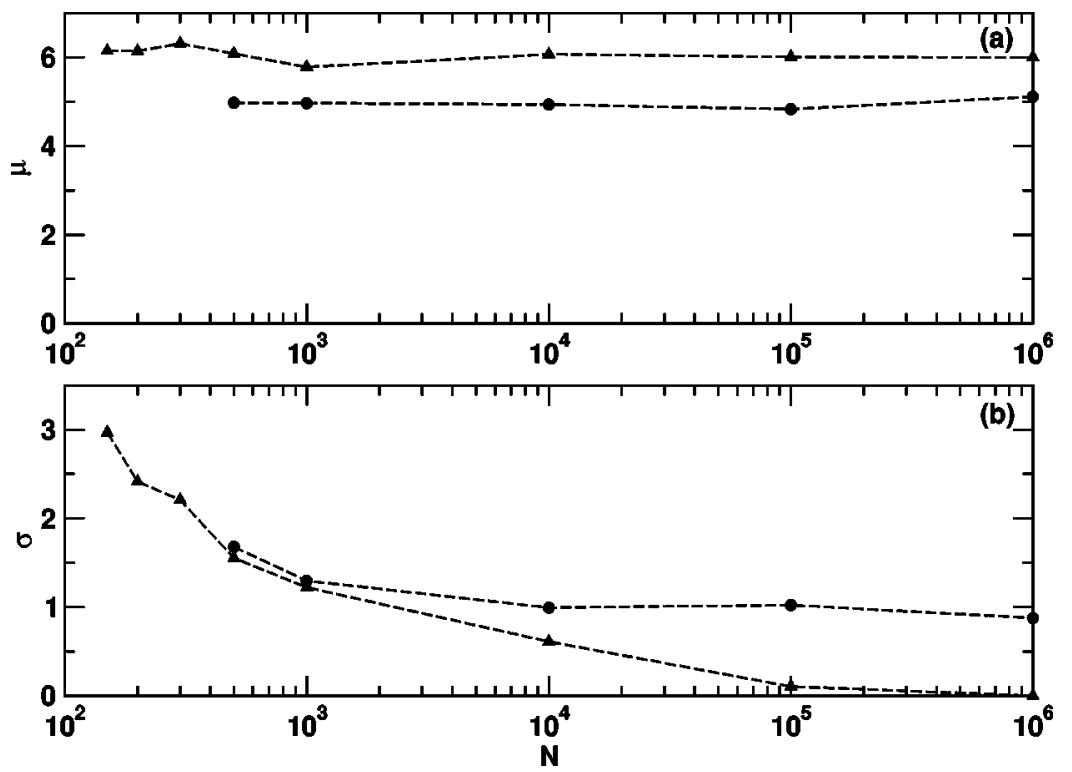

FIG. 15. (a) Variation of the mean value $\mu$ with $N$, for $P 6$ (triangles) and the chaotic orbit (circles). They are independent of the number of cells in the cluster. (b) Variation of the standard deviation $\sigma$ for $P 6$ (triangles) and the chaotic orbit (circles). to record using patch clamping techniques. Other methods, using for example the Ca signal, could also be applied.

First, a burst can be defined as the signal between two intersections with a certain value of the membrane potential chosen in the silent phase, that is, between the values of the voltage in the unstable point FP2 and the stable point FP3. $V=-60 \mathrm{mV}$ has proved to be a good choice. We have named this method the intersection algorithm. In this case we must check that between these two intersections the cell shows at least one spike in order to avoid spurious bursts due to small noisy fluctuations(we measure one spike when the signal for the voltage membrane intersects a sufficiently high value, for example $V=-35 \mathrm{mV}$ ). Second, we can use the ISI time series to define a burst, in what we call the ISI algorithm. As we already mentioned, periodic orbits show $d>0$ when $N>N_{d}$, where $d$ is the distance between the extremes of both peaks in an ISI histogram. For $N>N_{d}$, we know that there are no significant values of ISI belonging to $\left[d_{\min }, d_{\max }\right]$ and, therefore, a burst will be finished whenever ISI $>\mathrm{ISC}_{c}, \mathrm{ISI}_{c}$ being any value that verifies $\operatorname{ISI}_{c} \in\left[d_{\text {min }}, d_{\text {max }}\right]$. For $N>N_{d}$, this method gives exactly the same results as the former one.

For chaotic orbits, in which $d \approx 0$ for all $N$, as well as for periodic orbits in the range $N<N_{d}$, the ISI algorithm proves to be of little use. However, by again making use of the ISI histograms it is possible to choose a critical value of the interspike interval ISI $_{c}$ to distinguish spikes from bursts. The histograms are continuous in the considered cases, but a good choice is to use the value of the ISI for which the histogram shows a minimum between both peaks. Of course, this method is only useful for $N>N_{h_{H}}$, that is, for values of $N$ for which the histograms still show two peaks. After several tests, we have seen that the results of this method are very similar to the ones given by the intersection algorithm, only differing by a few bursts per time series.

Figure 15(a) shows the variation with $N$ of the mean value $\mu$ of the NSB time series for $P 6$ and the chaotic orbit. Up to 200 bursts have been computed to calculate $\mu$ and $\sigma$. These quantities are calculated for values of $N<10^{6}$, limit in which the orbits can be supposed completely indistinguishable from the deterministic limit. The standard deviations $\sigma$ for both orbits are shown in Fig. 15(b). Surprisingly, the mean value of the periodic orbit does not depend on $N$, at least until $N$ is very small. Therefore, for low NSB, the effect of the noise pushing the trajectories downwards in the $V$-Ca plane, crossing the $z$ nullcline and making them enter prematurely the silent phase is balanced by the lengthening influence of the reinjection effect, and the net conclusion is that the mean value $\mu$ is maintained approximately constant. We have not plotted the value of the mean for $P 6$ when $N<150$ and $C h$ for $N<300$, because here the destruction of the bursts is so strong that the two methods give substantially different results. Furthermore, $N \approx 150$ is the limit of the Gaussian approximation of the stochastic process and we have already mentioned the slight changes in the results that this fact can produce. It is remarkable that the mean value for the chaotic orbit is also constant. It is approximately equal to 5, which is explained if we look at its location in the bifurcation diagram in Fig. 1, just by a $P 5$ orbit. The standard deviation for the periodic orbit is zero for $N \gtrsim 5 \times 10^{5}$, which means that for $N>5 \times 10^{5}$ the noise is not strong enough to vary the number of spikes in any burst, all of them showing six spikes. The chaotic orbit, however, starts with $\sigma \approx 0.9$ in the absence of noise, and it maintains this value until it reaches $N \approx 10^{4}$, where it also starts to grow.

It is widely known that when the number of cells is small, the bursts are destroyed and they are replaced by a more random spiking behavior. However, the variation of the mean values in Fig. 15(a) for orbits with low NSB does not seem to be consistent with this result. In order to clarify this fact, we have analyzed in detail the approach from $N=150$ to $N$ $=1$ for $P 6$. We have done it only from a qualitative point of view, as for very small $N$ the signals are so noisy that there is not a robust way to measure the number of spikes in each burst. For small values of $N$, we obtain very short silent phases. Furthermore, the signal between two spikes of the same burst starts to reach very low values of the voltage, sometimes they even get down to the silent phase (around $V=-60 \mathrm{mV}$ ) and go up again to make another spike. Therefore, the concept of burst disappears as these structures be- 


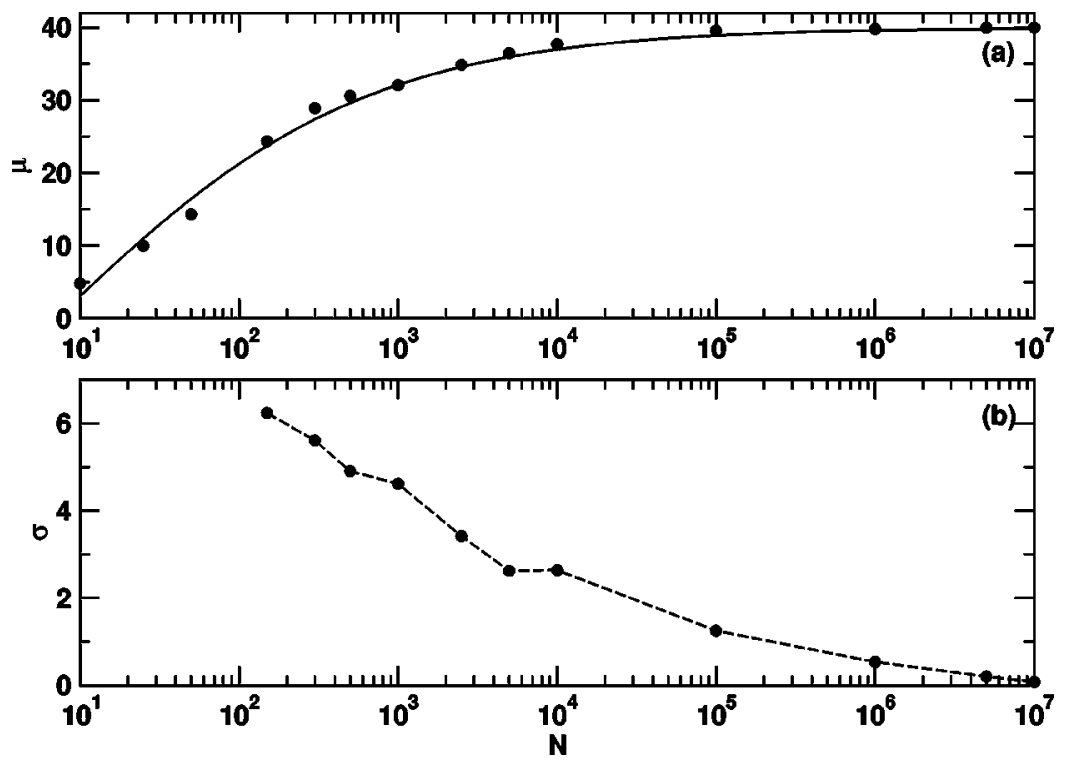

FIG. 16. (a) Variation of the mean value $\mu$ with $N$ for $P 40$. It decreases when $N$ decreases. The solid line represents the mathematical approximation. (b) Variation of the standard deviation $\sigma$ for $P 40$.

come a mixture of independent spikes, sometimes very close together and sometimes separated by a short silent phase. In conclusion, we do not have a gradual transition from bursts to spikes where $\mu$ tends slowly to 1 . What we see is a destruction of bursts produced by a growing noise that, eventually, is able to make the trajectory jump in any moment from the active to the silent phase and vice versa.

Figure 16 shows the variation of $\mu$ and $\sigma$ for a periodic orbit with high NSB, in particular for $P 40$. The results for $\sigma$ are very similar to those obtained for $P 6$ and $C h$ in Fig. 15, with the difference that the standard deviation reaches higher values and starts to grow for higher $N$ (we have not plotted the values of $\sigma$ for $N<150$ because, due to the inapplicability of the Gaussian approximation for such small $N$, they did not fit very accurately with the rest of the points). However, the variation of $\mu$ is totally different from that shown by $P 6$, as it clearly decreases when $N$ decreases. The reason for this opposite behavior is the already mentioned inexistence of the reinjection effect to balance the premature death due to the noisy escaping from the limit cycle basin. In this case only the first mechanism to show extra-long bursts is possible and eventually a few bursts show more spikes than the deterministic case. However, we already mentioned that this is a rare phenomenon, which cannot compensate the shortening effect of the noise. Furthermore, it is remarkable that the variation of the mean value $\mu$ with $N$ for $P 40$ can be described very precisely by the following expression:

$$
\mu=\mu_{D e t}[\tanh (a \ln N+b)]=\mu_{D e t}\left(\frac{A N^{B}-1}{A N^{B}+1}\right),
$$

where $\mu_{\text {Det }}$ is the NSB of the deterministic signal. Making use of genetic algorithm techniques, we have obtained that $A=0.42$ and $B=0.45$. The same expression obtained by least squares fitting, gives $A=0.37 \pm 0.04$ and $B$ $=0.52 \pm 0.02$. Both fittings show a correlation coefficient of $r=0.995$. We can see that the exponent of $N$ is very close to 0.5 . Furthermore, the mathematical fittings for other orbits $(P 30, P 20$, and $P 10)$ also show $B \approx 0.5$. There- fore, we obtain another example of the existence of a general dependence that relates the dynamics of our system with the square root of the number of cells $\sqrt{N}$.

\section{CONCLUSIONS}

In this work we have studied a mathematical model of the electrophysiological behavior of the pancreatic $\beta$ cell. Using this model as a paradigm of a bursting cell, we have focused our attention on the transition from the deterministic limit of large number of cells in an intact islet to the very stochastic behavior of an isolated cell. As mentioned in the Introduction, there are discrepancies to the hypothesis defended in this paper (the so-called channel sharing) to explain the different behavior shown by intact islets and isolated cells. However, the signals for the membrane potential obtained from the model appear quite similar to the actual signals obtained by the patch clamping techniques [31,32]. Furthermore, our analysis explores a stochastic regime that is not based on the simple addition of a noisy factor to the equations, but is one of the few examples that describes biological noise in terms of the underlying processes that generate the noise. For these reasons, we decided to use it to examine which tools are the best to obtain information from a bursting signal measured in a very noisy environment.

We started by analyzing briefly the main features of the deterministic model, and we have shown that depending on several parameters it can present continuous spiking, bursts with no spikes, and a wide range of bursts containing from very few to many spikes per burst. These signals could be both periodic and chaotic, being therefore a perfect environment to analyze how noise can blur and finally destroy the main properties of a signal. The characteristic period-adding transitions were previously described by Mosekilde et al. [33].

By making use of the Lyapunov exponents, we have seen that bursts with many spikes are much more robust under noise than those with few spikes. It should be remarked that bursts with many spikes show negative Lyapunov exponents 
even for very strong noise. The bursts that appear in real data are of this type, and this fact makes the task of calculating the Lyapunov exponents of experimental recordings very promising. Furthermore, we show that when $N$ decreases all Lyapunov exponents seem to grow more or less at the same rate, which is an important result because it means that they will maintain their relation even under strong noise.

In the development of this work we have paid special attention to the return maps and histograms of interspike time intervals and we have found out that they are fruitful sources of information. They can be easily obtained from real data with a very high accuracy, and applying clustering techniques they have proved to be extraordinarily useful to distinguish periodic from aperiodic orbits. Furthermore, we have used them to analyze the process of destruction of bursts when the cell clusters become very small and the level of noise reaches very high values.

Finally, we have studied the time series of the number of spikes in each burst for different types of orbits. In particular, we have explained why the mean of the NSB time series decreases when $N$ decreases for orbits with large number of spikes per burst, while in the cases in which this number is small the mean seems to be independent of $N$.

Throughout the paper, we have presented several mathematical expressions that fit our computational results with a very high accuracy. The dependence of all magnitudes on the square root of the number of cells $\sqrt{N}$ shows the existence of a deep relation between the source of the noise and the membrane potential signals. In some sense, the microscopic channel opening and closing processes are intimately connected with the macroscopic behavior of the cell. Furthermore, a direct consequenceof this fact could be a universal dependence of the main characteristics of the membrane potential on the size of the cellular cluster. If we suppose that the cells are located filling up the totality of the islets of Langerhans, and the islets are taken approximately spherical, we obtain that

$$
N \propto V \propto r^{3} \Rightarrow \text { Cellular properties } \propto \sqrt{V} \propto r^{3 / 2},
$$

where $V$ is the volume of the islet of Langerhans and $r$ its average radius. However, if we suppose that most $\beta$ cells are located in the surface of the islets, then a better geometrical approximation could be the following:

$$
N \propto S \propto r^{2} \Rightarrow \text { Cellular properties } \propto \sqrt{S} \propto r,
$$

where $S$ is the surface of the islet. In order to cast light on this subject, the development of the research about the dependence of the electrical properties of the cells on the geometry of the clusters seems to be a promising task.

In summary, it would be of significant interest to apply the tools developed in the present paper to experimental data obtained by patch clamping techniques. This way, we would be able to obtain valuable information about the behavior of clusters of pancreatic $\beta$ cells.

\section{ACKNOWLEDGMENTS}

We would like to thank Olga V. Sosnovtseva, Dmitry Postnov, Juan A. Almendral, Francesco d'Ovidio, Silvia de
Monte, Paul M. Diderichsen, Susanna Manrubia, and Sven Göpel for the fruitful talks we have had with them during the development of this research. This work has been supported by the Spanish Ministry of Science and Technology under Project Nos. BFM2000-0967 and BFM2003-03081 by a scholarship from the Spanish Ministry of Foreign Affaires (2001), and by Universidad Rey Juan Carlos under Project Nos. PGRAL-2001-02, PIGE-02-04, and GCO-2003-16. J.A. acknowledges support from the Danish Natural Science Foundation.

\section{APPENDIX}

The $\beta$-cell model studied in this work was first presented by Sherman et al. in Ref. [11]. The three differential equations for the deterministic model are the following:

$$
\begin{gathered}
C_{m} \frac{d V}{d t}=-\bar{g}_{\mathrm{K}} n\left(V-V_{\mathrm{K}}\right)-\bar{g}_{C a} m_{\infty}(V) h(V)\left(V-V_{\mathrm{Ca}}\right) \\
-\bar{g}_{\mathrm{KCa}} p\left(V-V_{\mathrm{K}}\right), \\
\frac{d n}{d t}=\lambda\left[\frac{n_{\infty}(V)-n}{\tau_{n}(V)}\right], \\
\frac{d \mathrm{Ca}}{d t}=f\left[-\alpha I_{\mathrm{Ca}}-k_{\mathrm{Ca}} \mathrm{Ca}\right],
\end{gathered}
$$

where

$$
p=\frac{\mathrm{Ca}}{\mathrm{Ca}+\mathrm{K}_{d}} \text { in the deterministic regime, }
$$

and

$$
p=\frac{n_{o}}{n_{o}+n_{c}} \text { in the stochastic regime. }
$$

Furthermore

$$
\begin{gathered}
m_{\infty}(V)=\left[1+\exp \left\{\frac{V_{m}-V}{S_{m}}\right\}\right]^{-1}, \\
h(V)=\left[1+\exp \left\{\frac{V-V_{h}}{S_{h}}\right\}\right]^{-1}, \\
n_{\infty}(V)=\left[1+\exp \left\{\frac{V_{n}-V}{S_{n}}\right\}\right]^{-1}, \\
\tau_{n}(V)=c\left[\exp \left\{\frac{V-\bar{V}}{S_{a}}\right\}+\exp \left\{\frac{-(V-\bar{V})}{S_{b}}\right\}\right]^{-1}, \\
\alpha=\frac{1}{2 V_{\text {Cell }} F} .
\end{gathered}
$$

The parameter that we have been varying is $V_{\text {Ca. }}$ Other parameters are the cell volume $V_{\text {Cell }}=1150 \mu^{3}$ (cell radius $R=6.5 \mu \mathrm{m}$ ), the total membrane capacitance $C_{m}=5310 \mathrm{fF}$, 
$\bar{g}_{\mathrm{K}}=2500 \mathrm{pS}, V_{\mathrm{K}}=-75 \mathrm{mV}, \bar{g}_{\mathrm{Ca}}=1400 \mathrm{pS}, \bar{g}_{\mathrm{KCa}}=30000 \mathrm{pS}$, $\mathrm{K}_{d}=100 \mu \mathrm{M}, \lambda=1.7, f=0.001, k_{\mathrm{Ca}}=0.03 \mathrm{~ms}^{-1}, V_{m}=4 \mathrm{mV}$, $S_{m}=14 \mathrm{mV}, V_{h}=-10 \mathrm{mV}, S_{h}=10 \mathrm{mV}, V_{n}=-15 \mathrm{mV}, S_{n}$ $=5.6 \mathrm{mV}, S_{a}=65 \mathrm{mV}, S_{b}=20 \mathrm{mV}, \quad c=60 \mathrm{~ms}$, and $\bar{V}=$ $-75 \mathrm{mV} . F=96487 \mathrm{C} / \mathrm{mM}$ is the Faraday constant. $\bar{g}_{\mathrm{K}}, \bar{g}_{\mathrm{Ca}}$ and $\bar{g}_{\mathrm{KCa}}$ are the maximal conductances. $\lambda$ is similar to the temperature in the Hodgkin-Huxley model and is nondimensional. $f$ is the fraction of free calcium in the cell. $\alpha$ is a unit conversion factor to change current into concentration/time. $k_{\mathrm{Ca}}$ is the net $\mathrm{Ca}^{2+}$ removal rate. Finally, $n_{o}$ and $n_{c}$ represent the number of open and closed channels in the stochastic regime.
[1] X. Wang and J. Rinzel, in Oscillatory and Bursting Properties of Neurons, The Handbook of Brain Theory and Neural Networks, edited by M. Arbib (MIT Press, Cambridge, MA, 1995).

[2] S. J. H. Ashcroft, J. M. Bassett, and P. J. Randle, Diabetes 21, 538 (1981).

[3] H. P. Meissner, J. Physiol. Paris 21, 757 (1976).

[4] H. P. Meissner and H. Schmelz, Pfluegers Arch. 351, 195 (1974).

[5] R. M. Santos, L. M. Rosario, A. Nadal, J. Garcia- Sancho, B. Soria, and M. Valdeolmillos, Pfluegers Arch. 418, 417 (1991).

[6] G. T. Eddlestone, A. Gonçalves, J. A. Bangham, and E. Rojas, J. Membr. Biol. 77, 1 (1984).

[7] P. Meda, I. Atwater, A. Gonçalves, J. A. Bangham, L. Orci, and E. Rojas, Q. J. Exp. Physiol. 69, 719 (1984).

[8] P. Smolen, J. Rinzel, and A. Sherman, Biophys. J. 64, 1668 (1993).

[9] D. Mears, N. F. Sheppard, Jr., I. Atwater, and E. Rojas, J. Membr. Biol. 146, 163 (1995).

[10] P. Rorsman and G. Trube, J. Physiol. (London) 374, 531 (1986).

[11] A. Sherman, J. Rinzel, and J. Keizer, Biophys. J. 54, 411 (1988).

[12] T. R. Chay and H. S. Kang, Biophys. J. 54, 427 (1988).

[13] T. A. Kinard, G. de Vries, A. Sherman, and L. S. Satin, Biophys. J. 76, 1423 (1999).

[14] F. C. Jonkers, J. C. Jonas, P. Gilon, and J. C. Henquin, J. Physiol. (London) 520, 839 (1999).

[15] J. A. Bangham, P. A. Smith, and P. C. Croghan, in Modeling the Beta-cell Electrical Activity, edited by I. Atwater, E. Rojas, and B. Soria Biophysics of the Pancreatic $\beta$-cell (Plenum, New York, 1986), pp. 265-278.

[16] G. de Vries and A. Sherman, J. Theor. Biol. 207, 513 (2000).

[17] G. de Vries and A. Sherman, Bull. Math. Biol. 63, 371 (2001).

[18] J. H. E. Cartwright, Phys. Rev. E 62, 1149 (2000).

[19] C. D. E. Boschi and E. Louis, Phys. Rev. E 65, 012901 (2001).

[20] Y. Loewenstein, Y. Yaron, and H. Sompolinsky, Proc. Natl. Acad. Sci. U.S.A. 98, 8095 (2001).

[21] Y. Loewenstein and H. Sompolinsky, Phys. Rev. E 65, 051926 (2002).

[22] Y. J. Liu, A. Tengholm, E. Grapenhiesser, B. Hellman, and E. Gylfe, J. Physiol. (London) 508, 471 (1998).

[23] I. Atwater, C. M. Dawson, A. Scott, G. Eddlestone, and E. Rojas, in The Nature of the Oscillatory Behaviour in Electrical activity from Pancreatic $\beta$-cell, Biochemistry and Biophysics of the Pancreatic $\beta$-cell, Hormone and Metabolic Research
Supplement Series Vol. 10, edited by W. J. Malaisse and I. B. Täljedal (Georg Thieme Verlag, Stutgart, 1980), pp. 100-107.

[24] T. R. Chay and J. Keizer, Biophys. J. 42, 181 (1983).

[25] A. L. Hodgkin and A. F. Huxley, J. Physiol. (London) 117, 500 (1952).

[26] N. F. Rulkov, Phys. Rev. Lett. 86, 183 (2001).

[27] G. de Vries, Phys. Rev. E 64, 051914 (2001).

[28] N. F. Rulkov, Phys. Rev. E 65, 041922 (2002).

[29] K. Saermark, M. Moeller, U. Hintze, H. Moelgaard, P. E. Bloch Thomsen, H. Huikuri, T. Makikiallio, J. Levitan, and M. Lewkowicz, Fractals 8, 315 (2000).

[30] W. Ren, S. J. Hu, B. J. Zhang, F. Z. Wang, Y. F. Gong, and J. X. Xu, Int. J. Bifurcation Chaos Appl. Sci. Eng. 7, 1867 (1997).

[31] S. Göpel, T. Kanno, S. Barg, J. Galvanovskis, and P. Rorsman, J. Physiol. (London) 521, 717 (1999).

[32] S. Göpel, T. Kanno, S. Barg, L. Eliasson, J. Galvanovskis, E. Renström, and P. Rorsman, J. Gen. Physiol. 114, 759 (1999).

[33] E. Mosekilde, B. Lading, S. Yanchuk, and Y. Maistrenko, BioSystems 63, 3 (2001).

[34] Y. S. Fan and T. R. Chay, Biol. Cybern. 71, 417 (1994).

[35] D. R. Cox and H. D. Miller, The Theory of Stochastic Processes (Wiley, New York, 1965).

[36] A. Wolf, J. B. Swift, H. L. Swinney, and J. A. Vastano, Physica D 16, 285 (1985).

[37] M. T. Rosenstein, J. J. Collins, and C. J. De Luca, Physica D 65, 117 (1993).

[38] R. Hegger, H. Kantz, and T. Schreiber, Chaos 9, 413 (1999).

[39] T. Sauer, Phys. Rev. Lett. 72, 3811 (1994).

[40] N. B. Janson, A. N. Pavlov, A. B. Neiman, and V. S. Anishchenko, Phys. Rev. E 58, R4 (1998).

[41] A. N. Pavlov, O. V. Sosnovtseva, E. Mosekilde, and V. S. Anishchenko, Phys. Rev. E 61, 5033 (2000).

[42] A. N. Pavlov, O. V. Sosnovtseva, E. Mosekilde, and V. S. Anishchenko, Phys. Rev. E 63, 036205 (2001).

[43] B. Mandelbrot, Fractals, Form, Chance, and Dimension (Freeman, San Francisco, 1977).

[44] J. L. Hudson and J. C. Mankin, J. Chem. Phys. 74, 6171 (1981).

[45] R. H. Simoyi, A. Wolf, and H. L. Swinney, Phys. Rev. Lett. 49, 245 (1982).

[46] J. A. Hartigan, Clustering Algorithms, (Wiley, New York, 1975).

[47] J. A. Hartigan, Appl. Stat. 28, 100 (1979).

[48] V. N. Belykh, I. V. Belykh, M. Colding- Joergensen, and E. Mosekilde, Eur. J. Phys. 3, 205 (2000). 